Gesnerus 60 (2003) 25-61

\title{
Gesundheit statt Galanterie
}

Der Paradigmenwechsel in ärztlichen Schönheitsratgebern im Jahrhundert der Aufklärung*

Sabine Sander

\section{Summary}

The views of femininity and the attempts to standardise it, developed in the 18th century in philosophy, education, literature, theology, anthropology and medicine, have led to intense research in the last years. Such research normally starts with the Enlightenment debates in the second half of the 18th century, but there are interesting sources which allow the tracing of a development over a longer period and which had a wider contemporary readership than any scholarly treatise. These are the popular beauty manuals, which in the German-speaking area started to appear more frequently from the late 17th century on, composed chiefly by physicians who thereby hoped to profit from the growing book and healthcare market. The present article considers the approaches of physicians to female beauty, with special reference to the discontinuities caused by the Enlightenment.

\section{Zusammenfassung}

Die im 18. Jahrhundert von Philosophen, Pädagogen, Literaten, Theologen, Anthropologen und Medizinern entwickelten Anschauungen und Normierungsversuche von Weiblichkeit haben in den letzten Jahren zu intensiver Forschung angeregt, die üblicherweise erst in der zweiten Hälfte des 18. Jahrhunderts mit dem Beginn des aufklärerischen Diskurses ansetzt. Eine interessante Quellengruppe, die es erlaubt, eine längerfristige Entwicklung

* Der vorliegende Aufsatz ist die überarbeitete Version eines Vortrages anlässlich der Wiener Gespräche zur Sozialgeschichte der Medizin vom 11. November 2000.

Dr. Sabine Sander, Medizinhistorisches Institut der Universität Mainz, Am Pulverturm 13, D-55131 Mainz (sander@mail.uni-mainz.de). 
nachzuzeichnen, und die von Zeitgenossen breiter rezipiert wurde als alle wissenschaftlichen Abhandlungen, ist bisher nicht systematisch untersucht worden: gemeint sind die populären Schönheitsratgeber. Im deutschsprachigen Gebiet erschienen sie in dichterer Folge seit der zweiten Hälfte des 17. Jahrhunderts und wurden vornehmlich von Ärzten verfasst, die sich damit ein Segment des Buch- und Gesundheitsmarktes zu erschliessen suchten. Der vorliegende Beitrag untersucht den sich in diesen Ratgebern widerspiegelnden ärztlichen Umgang mit der weiblichen Schönheit, mit besonderem Augenmerk auf die von der Aufklärung verursachten Brüche.

\section{Einleitung}

Die Geschichte der Aufklärung, der Frau und des Körpers sind drei intensiv bearbeitete Forschungsgebiete. Auch zur Schnittmenge dieser drei Themenkomplexe sind in jüngerer Zeit manche Studien vorgelegt worden. Als Beiträge zur sogenannten Gender-Forschung zielen sie zunehmend auf einen Vergleich der Geschlechter ab, wie etwa die Beiträge Londa Schiebingers zur Anatomie der Geschlechterdifferenz ${ }^{1}$. Viele dieser Arbeiten, namentlich diejenigen deutscher Provenienz, setzen in der zweiten Hälfte des 18. Jahrhunderts an, wodurch die Unterschiede zum Vorausgegangenen nicht klar erkennbar sind, manches sogar zu Unrecht als Innovation der Aufklärung deklariert wird ${ }^{2}$. Von den Monographien wäre vor allem Claudia Honeggers in drei Ausgaben erschienene und oft zitierte Ordnung der Geschlechter. Die Wissenschaften vom Menschen und das Weib $1750-1850^{3} \mathrm{zu}$ nennen. Aus französischen und deutschen Publikationen verschiedener Wissen(schaft)sgebiete präpariert Honegger die von Autoren der Aufklärungsepoche, nicht zuletzt Medizinern, entwickelte Anthropologie der Frau heraus. Auf die Schönheit als ein nach zeitgenössischer Auffassung konstitutives Element des Unterschiedes der Geschlechter, das auch in dem von ihr untersuchten Schrifttum eine gewisse Rolle spielte, geht sie allerdings nicht ein ${ }^{4}$.

Der Schönheit und Schönheitspflege waren und sind jenseits der GenderForschung verschiedene Monographien gewidmet. Auf breiter und hetero-

1 Vgl. Schiebinger 1995a, b und 2000b; vgl. auch Laqueur 1992, darin das Kapitel über «Die 〈Entdeckung〉 des Geschlechtsgegensatzes im Leib»,172-219.

2 Das hatte Brita Rang bereits 1986 in ihrer Auseinandersetzung mit Karin Hausens epochemachenden Aufsatz zur «Polarisierung der 〈Geschlechtscharaktere» (1976) kritisch angemerkt (vgl. Rang 1986, besonders S. 198), doch hat sich seither nichts geändert.

3 Honegger 1991/1992/1996.

4 Vgl. Honegger 1991, 175. 
gener Quellengrundlage ${ }^{5}$ nehmen sie zumeist grosse Zeiträume, vom alten Ägypten bis ins 20. Jahrhundert, in den Blick. Was die frühe Neuzeit angeht, beziehen sie sich vor allem auf die italienische Renaissance als eine frühe Blütezeit der Körperkultur, im übrigen aber auf Frankreich, das sich anschliessend zur Leitkultur entwickelte, und auf England ${ }^{6}$. Zu einer Art Klassiker hat sich aufgrund ihrer Materialfülle die in den letzten drei Jahrzehnten mehrfach aufgelegte Monographie Fashions in Makeup, from Ancient to Modern Times des Maskenbildners Richard Corson entwickelt ${ }^{7}$. Die Rolle der Ärzte auf dem Schönheitssektor wird in diesen Untersuchungen - zumal für die Zeit vor 1750 - üblicherweise nicht näher beleuchtet. Auch nicht in Arthur Marwicks Beauty in History ${ }^{8}$. Bei Philippe Perrot, der in Le travail des apparences ${ }^{9}$ das 18 . Jahrhundert in toto abhandelt, tauchen Ärzte erst im Zusammenhang mit den um die Jahrhundertmitte einsetzenden aufklärerischen Bestrebungen auf. Im übrigen stellt Perrot vornehmlich die für den Hof von Versailles und den französischen Adel bezeugten Praktiken zur Herstellung von Schönheit dar, so dass sich seine Ergebnisse nur partiell auf Deutschland übertragen lassen ${ }^{10}$.

Im Unterschied dazu soll hier mit einem spezieller medizinhistorischen Interesse nach den Ärzten als Fachleuten für Schönheit gefragt werden. Quellenbasis sind jene Ratgeber, mittels deren Ärzte ${ }^{11}$ ihr Wissen, aber auch ihr Frauenbild publik machten und in die Öffentlichkeit hineinwirkten ${ }^{12}$.

5 Bildliche und literarische Quellen stehen oft im Mittelpunkt des Interesses.

6 Letzteres aus sprachlichen Gründen, wegen des Quellenzugangs englischsprachiger Forscher.

7200 der 600 Seiten starken Monographie beziehen sich auf die frühe Neuzeit, und zwar auf französische und englische Verhältnisse; dazu bietet sie reiches, allerdings nur schwarz-weiss wiedergegebenes Bildmaterial. Vgl. Corson (1972, 1981, 1989 u.) 1997. Ähnlich angelegte Überblicke, aber weniger ausführlich und quellengesättigt, stammen von Angeloglou 1970, Wykes-Joyce 1961 und Pinset/Deslandres 1960. Vgl. auch die neuere, durch ihre gefällige Aufmachung mit zahlreichen farbigen Abbildungen für breitere Leserschichten reizvolle Zusammenstellung der Journalistin Renate Lohse-Jasper 2000. - Speziell zur Geschichte der Schönheitschirurgie, vor allem des 19. und 20. Jahrhunderts, Gilman 1999 und 2001.

8 Marwick 1988.

9 Perrot 1984.

10 Das Gleiche gilt für andere französische Untersuchungen wie Vigarello 1988.

11 Soweit feststellbar, geht der überwiegende Teil der oft anonym oder anonymisiert erschienenen Publikationen auf Ärzte zurück. Teils lassen sich die Verfasser namentlich ermitteln, teils ergeben sich aus den Texten Hinweise auf ärztliche Autorschaft. Allerdings gilt für die gesamte Untersuchungszeit, dass jeder entsprechend motivierte Nicht-Arzt prinzipiell in der Lage war, Bücher oder Artikel zu schreiben, die sich von ärztlichen nicht weiter unterschieden.

12 Diese Untersuchung der ärztlichen «Rollenindoktrination» gehört nach Gerda Lerners dreigliedrigem System zur untersten Stufe oder bildet einen ersten Schritt hin zu einer historischen Frauenforschung. Der zweite Schritt wäre eine Erforschung der Frauenkultur und des Frauenbewusstseins, im dritten Schritt, einer Vergleichsstudie, wäre das Spannungs- 
Als eigenständige Schriften erschienen sie im deutschsprachigen Raum seit der zweiten Hälfte des 17. Jahrhunderts. So ist der Untersuchungszeitraum gegenüber den zahlreichen, vor allem in Aufsatzform vorliegenden Studien zu den aufklärerischen Diskursen der zweiten Hälfte des 18. Jahrhunderts erweitert, so dass der Wandel im Denkstil und ärztlichen Selbstverständnis klarer hervortritt. Da die Verfasser allesamt Vertreter des männlichen Geschlechts, ihre Objekte und Adressaten vorwiegend Frauen waren, wird hier folglich «his story» und nur beschränkt «her story» berichtet. Die fraglichen Schriften sind in der älteren Medizinhistoriograhie wegen ihres Adressatenkreises und ihrer mehr oder minder ausgeprägten Volkstümlichkeit wenig beachtet worden ${ }^{13}$, obwohl doch gerade diese Besonderheiten ihre breitere zeitgenössische Rezeption ermöglichten. Allein schon angesichts ihrer Zahl ${ }^{14}$ beschränke ich mich aus forschungspraktischen Gründen auf das deutschsprachige Gebiet. Beabsichtigt ist eine quellennahe Untersuchung, die strenger systematisch vorgeht als die chronologisch aufgebauten Arbeiten. Inhaltliche Schwerpunkte liegen auf der ärztlichen Legitimation der Beschäftigung mit Schönheitsfragen, auf den von Ärzten vermittelten Idealen äusserer und innerer (körperlicher und moralischer) weiblicher Schönheit ${ }^{15}$, ihrer Bewertung der Schminke und des Schminkens und damit verbunden auf dem Paradigmenwechsel der Schönheitsmedizin im Zeichen von Aufklärung und Naturismus ${ }^{16}$.

verhältnis der männlichen und weiblichen Kulturen auszuloten. Vgl. Lerner 1989, 348f. - Die Frauen- und Gender-Forschung rekapitulieren neuerdings z.B. Schwarzkopf u.a. 2002 oder Daniel 2001, 313-330. Zum Gender-Aspekt speziell für die Fächerkombination Geschichte der Medizin, Naturwissenschaft und Technik vgl. den von Meinel/Renneberg 1996 herausgegebenen Sammelband, der in medizingeschichtlicher Hinsicht jedoch fast ausschliesslich auf die Rolle von Frauen als Heilerinnen bzw. Wissenschaftlerinnen eingeht; siehe darin insbesondere den Beitrag von Bleker.

13 Vgl. Sander 2002a.

14 Ein Überblick über diese Ratgeberliteratur ebenfalls bei Sander 2002a. - Ärzte der frühen Neuzeit verfassten nicht nur monographische Schriften, sondern publizierten auch in den im 18. Jahrhundert aufkommenden populären Zeitschriften, die ich im folgenden nur am Rande berücksichtige.

15 Eine Gegenüberstellung zu den Entwürfen von Männlichkeit wäre zwar wünschenswert, doch weil Männer im allgemeinen nicht Gegenstand der hier untersuchten Quellen waren, hiesse das im Rahmen dieses Aufsatzes, sich in einem Randgebiet zu verlieren. Vor den fragwürdigen Pauschalisierungen eines George L. Mosse in seiner Vorgeschichte der «modernen Männlichkeit» sei allerdings gewarnt, vgl. Mosse 1997, 9-25.

16 Andere Schwerpunkte setzen deutsche Forschungsarbeiten, die sich mit einem je unterschiedlichen Teil der hier interessierenden Schriften beschäftigt haben, wie die pharmaziegeschichtliche Dissertation von Gabriele Simon 1983 oder die germanistische von Gesa Dane 1994. Susanne Pellatz' Doktorarbeit liegen ausschliesslich pädagogische Schriften im engeren Sinne zugrunde, daher finden sich in ihr nur wenige Bemerkungen zu Hygiene und Moral um 1800, vgl. Pellatz 1999, 71-74. 


\section{Zur Legitimation ärztlicher Beschäftigung mit der Schönheit}

Zunächst mag man sich fragen, inwieweit sich die Zuständigkeit für weibliche Schönheit überhaupt mit dem Selbstverständnis eines Arztes, mit seiner Zugehörigkeit zum Seriosität beanspruchenden Gelehrtenstand vereinbaren liess, zumal in einer noch stark religiös fundierten Gesellschaft. Und tatsächlich sahen sich Ärzte unter einen gewissen Legitimationszwang gesetzt, wenn sie als «Galanterieärzte» ${ }^{17}$ tätig waren: «Hier fragt sichs nun nicht unbillig, ob einem Medico zukomme eine solche Kunst zu lehren und öffentlich davon zu schreiben? $\gg^{18}$ So formulierte es Tobias Vogel, gräflicher Leib- und Hofarzt im thüringischen Greiz, der 1690 mit seinem umfangreichen Curieusen Haut-Diener, einem landessprachigen kosmetisch-dermatologischen Kompendium, einen Trendsetter der Schönheitsmedizin auf den Markt brachte. Er beantwortet die Frage selbst ausführlich, unter Rückgriff auf weltliche und geistliche Autoritäten. In Anlehnung an Galen unterschied er zwischen «Cosmetica» einerseits, die gleichsam eine «Reinmachungskunst» sei, und Putz oder Schminke andererseits, mit denen Missbrauch getrieben würde und die den Beigeschmack des «Hurenmäßigen» hätten. Erstere erhalte die natürliche Schönheit oder stelle die verlorene Schönheit wieder her, letztere versuche, eine nie dagewesene Schönheit künstlich herzustellen. Erstere sei ein Stück der Arzneikunst, letztere aber sei verwerflich und daher eigentlich nicht Sache des Arztes.

Doch hob der Autor sogleich die zuvor getroffene scharfe Zweiteilung wieder auf. Galen selbst habe zugegeben, dass der Übergang zwischen Kosmetik und Putz fliessend sei. Und Avicenna habe sich nicht gescheut, Schönheitsrezepte und -regeln zu lehren. Ausserdem könne ein Arzt, der ein «vornehmes Frauenzimmer» bediene, sich nicht allein auf die von moralischen Bedenken freie «Cosmetica» beschränken, sondern müsse tun, was gefordert werde. Hier wird die noch schwache Stellung des Arztes gegenüber Patienten aus der Oberschicht ersichtlich. Neben fachlichen und sozialen bzw. marktstrategischen, sozusagen weltlichen Argumenten werden auch christliche angeführt: Der Mensch sei nicht um seiner selbst willen da, die Rücksicht auf den Nebenmenschen erfordere einen erfreulichen Anblick, zumal im Schöpfungsplan der Mensch als Abdruck des göttlichen Bildnisses gedacht sei. Könnten bisweilen doch Ehen gerettet werden, indem die Frau für ihre Schönheit sorge. Ausserdem hätten weder Heilige Schrift noch die

17 Vgl. den Buchtitel Galanterie-Artzt des späteren Professors der Medizin in Königsberg, Woyt $1699 / 1710$.

18 Vogel 1690,370, zum Folgenden vgl. 370-374. 
Kirchenväter das Putzen und Schminken rundweg verdammt. Es solle aber mit Mass und Ziel betrieben werden, mit der Ehrbarkeit vereinbar sein. In diesem Sinne hätten im übrigen auch schon Cicero und Ovid gesprochen. Durch diese Rechtfertigungen abgesichert, konnte der Autor dazu übergehen, seine Schönheitsmittel vor den interessierten Leserinnen ${ }^{19}$ auszubreiten.

Diese Legitimation findet sich in unterschiedlicher Ausführlichkeit in mehreren einschlägigen, überwiegend aus Rezeptsammlungen bestehenden Publikationen aus der ersten Hälfte des 18. Jahrhunderts, so im 1704 erfolgten, nur wenig bearbeiteten und nicht-autorisierten Nachdruck eines Anonymus unter dem Titel Neu-entdeckte Geheimnisse von der Schönheit der Damen, der seinerseits wenig später Christoph Hellwig als Kompilationsvorlage für seine Heimligkeiten des Frauenzimmers diente ${ }^{20}$; beide Druckschriften wurden in der ersten Jahrhunderthälfte noch mehrfach aufgelegt.

Ein anderer (anonymisierter) Autor der frühen Zeit, der gegen Hoffart und Eitelkeit eiferte und sich auf die Heilige Schrift berief, betonte ausserdem seinen beruflichen Rang, dass er ein Medicus und kein Tüncher sei, sowie seine persönliche Integrität, dass er seine Arcana der Leserschaft nur in redlicher Absicht eröffne und an den Galanterien nicht reich werden wolle ${ }^{21}$. Damit hatte er der Pflicht Genüge getan und konnte seine Rezepte folgen lassen.

Ärzte in der zweiten Jahrhunderthälfte hatten angesichts des inhaltlichen Wandels der Schönheitsschriften solche Rechtfertigungen nicht mehr nötig. Vom Galanteriearzt alten Stils distanzierten sie sich explizit. Ein solcher galt ihnen als Charlatan und Quacksalber. Auch durfte sich ein auf der Höhe der Zeit befindender Arzt nicht mehr mit der Eröffnung von Arcana brüsten. Erwähnt sei hier nur der aus dem Französischen übersetzte Arzt des Frauenzimmers von 1773. Der nicht namentlich genannte französische Autor hat in der Originalausgabe seinen Ausführungen über Körper, gesunde Lebensweise und Krankheiten der Frau in einem Anhang der Gesundheit nicht

19 Deren Interesse lässt sich schon allein aufgrund der Bedeutung der über 250 fürstlichen Höfe im deutschen Reich nach dem Ende des 30jährigen Krieges und der damaligen Orientierung an der französischen Mode und Kultur infolge des deutschen Entwicklungsrückstandes unterstellen.

20 Anonym 1704, spätere, allerdings erheblich gekürzte Ausgaben unter dem Titel «LeibDiener der Schönheit»: 1747 und 1751; Hellwig 1715, spätere Ausgaben: 1719, 1720, 1725 und 1734. Die Schriften Vogels, des Anonymus oder Hellwigs benutzte noch viel später der nichtärztliche Vielschreiber Kritzinger, der seine Variante 1777 und 1788 unter dem die Zeitgenossen zweifellos bewusst irreführenden Pseudonym (Wilhelm) Tissot herausbrachte.

21 Vgl. D. F. 1715, 8-11. 
abträgliche Schönheitsmittel ausdrücklich nur deshalb angefügt, weil Leserinnen dergleichen von einem Gesundheitsratgeber nun einmal erwarteten; andere Autoren beriefen sich bei solchen Anhängen auf das Drängen ihres Verlegers. Der deutsche, ebenfalls ungenannte Übersetzer war in seiner kritischen Distanz rigoroser und erklärte dazu:

Diesen Anhang habe ich weggelassen; theils weil ich die Deutschen Frauenzimmer für zu einsichtsvoll, und über den falschen Schein zu erhaben glaubte, als dass sie die natürliche Gestalt verbessern, oder vielmehr verschlimmern wollten; theils weil ich es für einen Arzt unanständig achtete, der Eitelkeit ihr Schminkdiener zu sein. ${ }^{22}$

Hier hat sich das ärztliche Selbstverständnis sehr deutlich gewandelt. Die Generation der in der zweiten Jahrhunderthälfte, vor allem in den letzten drei Jahrzehnten des 18. Jahrhunderts publizierenden Aufklärer sah ihre Aufgabe nicht mehr darin, Rezepturen für Schminke zusammenzustellen, wie sich aufklärerische Ärzte auch weigerten, Rezepte für Heilmittel den Lesern in die Hand zu geben ${ }^{23}$. Die Selbstbehandlung der Patienten schien ihnen grundsätzlich nicht mehr wünschenswert, denn sie beanspruchten nun, die einzigen Sachverständigen und Behandler zu sein. Darüber hinaus erschien ihnen die Kosmetik im besonderen unvereinbar mit ihrem ärztlichen Berufsethos. Wenn sie sich ihr weiterhin widmeten, dann in allererster Linie als deren Kritiker. Als wesentliche Motive ihrer Beschäftigung mit der Schminkfrage führten sie jetzt die Suche nach der ungeschminkten Wahrheit sowie patriotische Sorge um Wohl und Gesundheit der weiblichen Bevölkerung an. Da die Verhütung von Krankheiten neben der rein kurativen Tätigkeit ohnehin den Kern des Arztberufes ausmachte, bestand für Ärzte als kritische Aufklärer in kosmetischen Fragen keine besondere Notwendigkeit mehr, sich gegenüber der Leserschaft zu legitimieren. Hatten Mediziner anfänglich gemeint, die Vereinbarkeit ihrer speziellen ärztlichen und schriftstellerischen Arbeit mit der Moral nachweisen zu müssen, so war es im späten 18. Jahrhundert die ärztliche Moral selbst, die sie zur Beschäftigung mit der Kosmetik veranlasste ${ }^{24}$.

22 Anonym 1773, Vorwort des Verfassers (ohne Paginierung).

23 So erklärte zum Beispiel der Arzt und Publizist Ernst Baldinger: «Ich habe es mir zum Gesetz gemacht, in meinen Lehren gerade da aufzuhören, wo in anderen Schriften die Recepte folgen» (Baldinger 1768, 52).

24 Den neuen Ton schlugen erstmals Oelßner und Struve, beide 1754, an. Siehe die gereimte Vorrede bei Oelßner «An den Leser» (ohne Paginierung); Struve, Vorrede. 


\section{Zum männlichen Ideal weiblicher Schönheit}

Wie aber veränderten sich im kulturellen Wandel von Barock und Rokoko zur Aufklärung, von aristokratisch-höfischen Leitbildern zu bürgerlichen die Vorstellungen vom idealen weiblichen Körper? ${ }^{25}$ Die Idealvorstellungen lassen sich zum Teil aus den erhofften Wirkungen der kosmetischen Mittel erschliessen. Danach stand die makellos feine, glatte und glänzende Haut, blass, mit einem Hauch von Röte, im Zentrum der Bemühungen um gutes Aussehen. Darüber hinaus aber wurde in vielen Schriften die Frage nach der Schönheit explizit aufgegriffen, wohl nicht zuletzt, um damit dem Leserinteresse entgegenzukommen. Grundsätzlich blieb man sich über die Zeiten hinweg weitgehend einig in der Überzeugung, dass sich das weibliche Geschlecht durch besondere Schönheit vor dem männlichen Geschlecht auszeichne, so dass nur ausnahmsweise und ganz am Rande männliche Schönheit überhaupt thematisiert wurde.

Als konstitutive Elemente weiblicher Schönheit und menschlicher Schönheit schlechthin galten noch vor allen Details Mittelmass in Grösse und Stärke des Leibes, Ebenmass, Proportioniertheit und Harmonie. Die Gesetze von Proportion und Harmonie zu ermitteln war seit der Antike ein Anliegen von Philosophen, Künstlern und Architekten ${ }^{26}$. Unter Berufung auf Albrecht Dürer, den in diesen Fragen massgeblichen deutschen Künstler, gab Vogel die idealen Verhältniszahlen aller Teile des Leibes für beide Geschlechter an, wobei ihn in diesem Zusammenhang zunächst der männliche Körper als Norm und Muster interessierte ${ }^{27}$. Aber nicht allein die Ordnung (nach Aristoteles), sondern erst Ordnung bzw. Proportion und Farbe (letzteres nach Galen) seien die entscheidenden Kategorien zur Bestimmung der Schönheit. Nach seiner mit vielen historischen Erläuterungen versehenen Diskussion der weiblichen Einzelelemente der Schönheit von Kopf bis Fuss gab Vogel ein aus dreissig Punkten bestehendes Ideal wörtlich wieder, das der französische (nicht-medizinische) Autor François de Grenailles ${ }^{28}$ Jahrzehnte zuvor in seinen Plaisirs des femmes zusammengestellt hatte, die seit 1653 unter dem Titel Frauenzimmer-Belustigungen in deutscher Übersetzung

25 Die Schönheitsvorstellungen werden in der Forschungsliteratur in der Regel knapp und summarisch anhand nicht-medizinischen Quellenmaterials, meist unter besonderer Berücksichtigung bildlicher Darstellungen behandelt. Vgl. vor allem Marwicks Beauty in History (1988).

26 Allgemein zum Schönheitsbegriff Most 1992 und Ritter 1971.

27 Vgl. Vogel 1690, 163f.; zum Folgenden vgl. Vogel 1690, 164-168. - Vgl. auch Verspohl 1998 und Ridder 1996.

28 Schreibweise des Eigennamens nach der Nouvelle Biographie Génerale (Audoin 1857), auf dem Titelblatt der deutschen Übersetzung dagegen: Greneille. 
vorlagen. Auch dieser Katalog ${ }^{29}$ geht von allgemeinen, den ganzen Körper betreffenden Merkmalen aus, um sodann, dem traditionellen topographischen Schema a capite ad calcem folgend, die als wesentlich erachteten Einzelmerkmale zu nennen. Unter der Vielzahl von Aspekten der Schönheit spielten Zierlichkeit, Kleinheit und Feinheit vieler Körperteile eine bedeutende Rolle: Die Augenbrauen sollten dünn und kurz, Mund und Zähne sowie die Ohren klein sein, ebenso die Füsse. Die Farben Weiss und $\operatorname{Rot}^{30}$, die Autoren immer wieder zu Vergleichen mit Milch und Blut oder Lilien und Rosen veranlassten, waren ebenfalls wichtig: weiss bzw. «röthlich weiß» galt als Idealfarbe der im übrigen makellosen Haut, des «helffenbeinernen» Halses, der «alabasternen» Brust, der schneeweissen Hände; korallenrot sollten die Lippen sein, rötlich aber auch die Ohren. Die Augenfarbe sollte schwarzbraun sein, die Haare kastanienbraun, fein und gelockt. Besonders das Merkmal weisse Haut, aber auch die Preziosen-Metaphorik machen deutlich, dass es sich um ein standesgebundenes Schönheitsideal handelte, das für die grosse Mehrheit der landwirtschaftlich tätigen und somit der Sonne ausgesetzten Frauen unerreichbar blieb. Die Röte könnte man als Garantin von Gesundheit und Jugendlichkeit deuten ${ }^{31}$, die ohnehin als Bestandteile oder Voraussetzungen körperlicher Attraktivität galten.

Vogels oben genannte Nachfolgeautoren sorgten für die weitere Verbreitung dieses Schönheitskataloges. Hellwig nahm eine geringfügige Modifikation vor und fügte einen 31. Punkt hinzu, indem er (an sachlich unpassender Stelle) das Ideal kastanienbrauner Haare nicht als ausschliessliches akzeptierte und wegen der individuellen Geschmacksunterschiede noch «schöne weisse oder gelbe» sowie «schwartze» Haare ergänzte ${ }^{32}$. An ebendiesem Detail, den Haarfarben, ist erkennbar, dass auf dem Umweg über Hellwigs mehrfach aufgelegtes populärmedizinisches Buch Grenailles' Schönheitskatalog in wichtige nicht-medizinische Werke Eingang fand: in das 1715 erstmals veröffentlichte, 1739 und 1773 erneut aufgelegte FrauenzimmerLexicon, von Gottlieb Siegmund Corvinus unter dem Pseudonym Ama-

29 Solche aufzählenden Schönheitsbeschreibungen hatten eine gewisse Tradition. In der schönen Literatur Frankreichs aus dem 16. Jahrhundert etwa gibt es ein Gedicht mit dem Titel «Dreiundzwanzig Schönheiten, welche eine schöne Frau besitzen soll» (nach Brockmeier 1988, 204), wobei die Zahl 23 - im Unterschied zur runden 30 - als allzu willkürlich gewählt erscheint.

30 Schon im biblischen Hohenlied Salomons heisst es, dort allerdings in bezug auf den Mann: «Mein Freund ist weiß und rot» (Hoheslied 5.10.).

$31 \mathrm{Vgl}$. Schuster 1993, 14f.

32 Hellwig, 1715, 26f., hier 27 (Anm. 5). Da rote Haare bekanntlich als unschön, ja sogar anstössig galten, fehlen sie auch in Hellwigs komplettierter Liste. 
ranthes herausgegeben, sowie in das Monumentalwerk des Leipziger Buchhändlers und Verlegers Johann Heinrich Zedler, das Universal Lexicon aller Wissenschaften und Künste, und zwar in den Artikel über die «Schönheit des Frauenzimmers». In beiden Fällen wurde (durch Zusammenfassung der die Haare betreffenden Punkte) die Liste der Schönheitsmerkmale wieder von 31 auf 30 reduziert ${ }^{33}$.

Diese runde Zahl, die Ordnung schaffende aufzählende Form waren zweifellos Ursache dafür, dass das Schönheitsideal später von Autoren populärmedizinscher Werke selbst in ihren inhaltlich neu ausgerichteten Schönheitsschriften übernommen wurde, von Gottlieb Oelßner 1754 in seine für Leser beiderlei Geschlechts gedachten modekritischen Ratgeber ${ }^{34}$, aber auch von dem Nicht-Arzt Friedrich A. Kritzinger unter dem Pseudonym Wilhelm Tissot in den Jahren 1777 und $1788^{35}$. Schliesslich treffen wir den alten Schönheitskatalog noch 1793 in der medizinischen Frauenzeitschrift Hygea an ${ }^{36}$. Im Unterschied zur älteren Zeit systematisierte der ungenannte Autor des betreffenden Artikels nicht mehr nach dem A-capite-ad-calcem-Schema, sondern er verwendete eine andere, ausgefeiltere Systematik, indem er das Dezimalsystem mit der seit alters beliebten Drei verband und zehn verschiedenen Eigenschaften jeweils drei Körperteile zuordnete ${ }^{37}$. Dabei behauptete sich Zierlichkeit mit den charakterisierenden Epitheta kurz, eng, fein und klein als zentrales Element weiblicher Körperästhetik. Sie wurde für Kopf, Nase, Ohren, Mund, Lippen, Zähne, Taille und Füsse, ausserdem auch für die Brustwarzen postuliert. Dick aber sollten ausser den früher schon erwähnten Armen auch Schenkel und Waden sein. Das alte Ideal weisser und roter Farben galt noch immer, ebenso das Ideal dunkler Augen.

Neben dem 30-Punkte-Katalog gab es im späten 18. Jahrhundert eine pädagogisch wirksamere Form der Präsentation des Schönheitsideals, nämlich die exemplarische Darstellung ${ }^{38}$. Ein Anonymus, der - im Unterschied zu den Autoren der ersten Jahrhunderthälfte - als typischer Aufklärer erzieherisch wirken wollte, bot seinen Leserinnen ein namentlich benanntes und damit (scheinbar) authentisches Mädchen als Identifikationsfigur an. Aus-

33 Vgl. Art. «Schönheit» 1715, 1757f.; Art. «Schönheit des Frauenzimmers» 1743, 822.

34 Oelßner 1754, 8f.

35 Tissot (= Kritzinger) 1788, 17f. Seine Liste enthält 31 Punkte wie bei Hellwig, aber nur eine vorbildliche Haarfarbe, nämlich Kastanienbraun; sein Zusatz an 31. Stelle lautet: «schöne Liniamente».

36 Hygea 1793, 2. Stück, 94.

37 Auch für solche Bündelungen zu Dreiergruppen gab es alte literarische Vorbilder (vgl. Brockmeier 1988, 205). Ohne nähere geographische oder historische Einordnung spricht auch Marwick, der deutsche Quellen nicht weiter berücksichtigt, von der «elaborate and very popular thirty, grouped in threes» (Marwick 1988, 68).

38 Vgl. zum Folgenden Anonym 1785, 39-42. 
serordentlich detailliert führte er am fiktiven Beispiel einer Magdalis seine Vorstellungen von weiblicher Schönheit aus, wozu ihn offensichtlich die gleichnamige lyrische Figur eines anakreontischen Gedichts seines Zeitgenossen Gleim inspiriert hatte. Obwohl er sonst immer wieder wie andere Autoren seiner Epoche - in Schillers Diktion möchte man sagen: sentimentalisch - die natürliche Schönheit der Landmädchen beschwor, denen nicht der Schminkpinsel, sondern gesunde Lebensweise die Rosenröte auf die Wangen male, ist seine Magdalis freilich doch eine Repräsentantin der gehobenen und verfeinerten städtischen Gesellschaft, an die sich die Kosmetikliteratur (meist implizit) generell wandte. An späterer Stelle des gleichen Buches stellte der Autor dann nochmals ins Allgemeine gewendet sein bzw. das zeitgenössische Ideal einer schönen Frau vor. Es unterscheidet sich, was das Äussere angeht, nicht nennenswert von dem alten Ideal Grenailles' ${ }^{39}$.

Insgesamt überrascht die Beständigkeit des Schönheitsideals in der hier untersuchten Literatur in einem Untersuchungszeitraum von rund 150 Jahren $^{40}$, und das in einer historischen Phase, die von den im allgemeinen sehr fortschrittsgläubigen Zeitgenossen selbst als eine des gewünschten und bejahten Umbruchs erlebt wurde. Mit der starken Gewichtung der Zierlichkeit, Kleinheit und Feinheit für die weibliche Schönheit stellte man implizit einen starken Kontrast zur Männlichkeit her ${ }^{41}$ und rückte zugleich den als

39 Die wenigen von Perrot aus zwei französischen Quellen der Jahrhundertmitte zitierten Schönheitsmerkmale stimmen mit den in deutschen Ratgebern genannten im wesentlichen überein. Vgl. Perrot 1984, 69f.

40 Es handelt sich um ein recht universelles Schönheitsideal, von dem sich die des Mittelalters und der italienischen Renaissance vornehmlich unterscheiden durch die Favorisierung blonder Haare, die sich bekanntlich schon in der römischen Antike besonderer Beliebtheit erfreut hatten; im Mittelalter schätzte man blaue Augen besonders (vgl. Marwick 1988, 68; ferner Corson 124f. und 227f.). Neben seiner Dauerhaftigkeit ist die geographische Verbreitung des Schönheitsideals auffallend, denn es findet sich mit nur geringfügiger Abweichung im ostjüdischen Volksliedgut, das Tamar Somogyi allerdings zeitlich nicht klar einordnet und differenziert. Darin kommen folgende Versatzstücke in unterschiedlicher Häufigkeit vor: lange, gelockte blonde Haare; schwarze Augen; rosarote Wangen und Lippen; weisse Zähne; eine schöne Taille; weisse Hände und kleine Füsse (vgl. Somogyi 1982, 99-103). - Diese Elemente finden sich auch in den «Idealen weiblicher Schönheit bei den Morgenländern», nur mussten die langen gelockten Haare schwarz sein. Die Grösse der Füsse spielte dort keine Rolle, dafür war die Beschaffenheit der Brüste und anderes, was der Orientalist in Rücksicht auf das Schamgefühl seiner deutschen Leser nicht ansprechen wollte, wichtig; auch wurde der Duft der arabischen Tradition entsprechend hoch bewertet (vgl. Hartmann 1798, besonders 45f., 121f., 132-135).

$41 \mathrm{Vgl}$.auch Soemmerrings Entwurf eines idealen weiblichen Skeletts, der «schönen Mainzerin» (neuerdings auf dem Titelblatt von Dumont u.a. 2002), das ganz vom Kontrast zum «typisch männlichen» Körper bestimmt war (Stolzenberg-Bader 1989, 795-801). Solche Kontrastierung der Geschlechter ist keineswegs selbstverständlich, wie das ostjüdische Beispiel zeigt, wo der Mann nicht gross und stark sein sollte, was als «gojisch» (nicht-jüdisch) galt, sondern zart und feingliedrig aufgrund der hohen Wertschätzung der Gelehrsamkeit, die beim Mann nicht nur einen Mangel an physischer Attraktivität, sondern sogar Missbildungen wett- 
schön deklarierten Frauentypus in die Nähe des Kindes ${ }^{42}$. Unterstrichen wurde dies mit der verstärkten Betonung rundlicher Körperformen, eines rundlichen Gesichts oder gar fetter Arme oder weisser, weicher, zarter und fleischiger Hände, auf denen keine Adern hervortreten dürfen, wobei sich die Vorstellung von Kinderarmen und -händen aufdrängt. Nur in der Forderung einer schlanken, aber ungeschnürten Taille und in der verstärkten Betonung der Reinlichkeit im Zusammenhang mit der äusseren Erscheinung deutete sich in der Ratgeberliteratur des späten 18. Jahrhunderts ein zunehmender Sinn für den Zusammenhang von Gesundheit und Schönheit an. Dabei war die ungeschnürte Taille der aus medizinischen Gründen geforderte männliche Tribut an die nun in den gesellschaftspolitischen und wissenschaftlichen Diskussionen in den Mittelpunkt gerückte Mutterrolle der Frau ${ }^{43}$.

\section{Äussere Schönheit - innere Schönheit}

Von unserem ältesten bis zum jüngsten Textzeugnis wurde auch von ärztlicher Seite nie ausschliesslich die äussere Schönheit besprochen. Tobias Vogel akzentuierte sogar gleich im Frontispiz seines Spiegels der menschlichen Schönheit Tugendhaftigkeit als innere Schönheit mit dem Sinnspruch «die Schönheit ist flüchtig, das Schmincken untüchtig, die Tugend Ziert richtig», was zur Zeit der Abfassung des Buches im späten 17. Jahrhundert auch ein Prophylaktikum gegen mögliche Kritik von klerikaler Seite gewesen sein $\mathrm{mag}^{44}$. Für Christoph Hellwig im frühen 18. Jahrhundert stimmten äusserliche und innerliche Schönheit idealerweise überein: «Ist demnach mein und aller

machen konnte (vgl. Somogyi 1982, 98f.); die gleichwohl auch bei den Ostjuden bestehende Polarität bzw. Komplementarität der Geschlechter lag darin, dass Frauen mit körperlicher Schönheit das Weltliche, Männer durch Intellektualität und Leistung das Geistige repräsentierten bzw. repräsentieren sollten.

42 Das venezianische Schönheitsideal des 16. Jahrhunderts hatte die Frau ebenfalls in die Nähe des Kindes gerückt, wenn auch statt der Zierlichkeit die haarlose und wie später noch die kindlich helle und weiche Haut bedeutsam gewesen war (vgl. Gadebusch Bondio 1996, 71). - Im späten 18. Jahrhundert sah etwa Soemmerring Kindnähe bei der Frau in Krankheitsanfälligkeit, Körperbau und Physiognomie gegeben (vgl. Stolzenberg-Bader 1989, 776-780). - Ein weibliches Gesicht, das sich dem Kindchenschema nähert, besitzt auch in heutigen Tests erhöhte Attraktivität für die Probanden (nach Richter 1999,120).

43 Aus Soemmerrings Arbeit Über die Wirkung der Schnürbrüste (1788) geht hervor, dass daneben die in der zweiten Hälfte des 18. Jahrhunderts an Kunstwerken der griechischen Antike erfolgende Geschmacksbildung einen Einfluss auf das Schönheitsideal auch in der Medizin ausübte. Vgl. Stolzenberg-Bader 1989, 799-808.

44 Zur englischen klerikalen Kosmetikkritik in der zweiten Hälfte des 17. Jahrhunderts vgl. das Kapitel «The Sin of Painting» bei Corson 1997, 152-155. 
redlichen Hertzen Wunsch, dass die Schönheit des Leibes mit der Schönheit des Gemüths, die äusserliche und innerliche Schönheit, fein miteinander mögen verknüpffet seyn und bleiben.» ${ }^{45}$ Erst die Tugenden brächten die äussere Schönheit voll zur Geltung und blieben als unvergängliche Eigenschaften erhalten, wenn die äussere Schönheit in Alter oder Krankheit verlorengehe. $\mathrm{Zu}$ den geschätztesten Tugenden gehörten zunächst besonders die christlichen, nämlich Gottesfurcht, Frömmigkeit, Freundlichkeit, Demut und Wahrheitsliebe, Einfalt, Keuschheit, Züchtigkeit, Schamhaftigkeit und «Stillschwiegenheit», erst in zweiter Linie die eher als bürgerliche Tugenden zu bezeichnenden wie Häuslichkeit, zurückgezogene Lebensweise und Fleiss.

Mit der allgemeinen Säkularisierung, die sich auch in der Diskussion von Schönheitsfragen beobachten lässt ${ }^{46}$, ging die Neigung zur Moralisierung der Schönheit aber keineswegs verloren - ganz im Gegenteil. Wegbereiter einer dezidiert moralisierenden Betrachtung waren die seit den 1720er Jahren auch in Deutschland nach englischem Vorbild eingeführten frühaufklärerischen Moralischen Wochenschriften ${ }^{47}$. Dort war sogar von der Nichtswürdigkeit der äusseren Schönheit die Rede ${ }^{48}$. Statt Schönheit wünschte man die Bildung von Gemüt, Seele und Herz, nur vereinzelt mehr Kenntnisse in den Wissenschaften ${ }^{49} .1756$ spöttelte zwar auch der Arzt Christian Tobias Ephraim Reinhard in seiner Satyrischen Abhandlung, der feinsten Zärtlichkeit der Haut sei mehr Verstand vorzuziehen ${ }^{50}$. Höher als Verstand aber bewerten ärztliche Aufklärer in der zweiten Hälfte des 18. Jahrhunderts allemal moralische Qualitäten, die in den Jahrzehnten stetig fortschreitender Verweltlichung nicht mehr aus dem Christentum abgeleitet wurden, sondern im Empfindsamkeitskult der Bildungsschicht wurzelten, dessen Inhalte und Ausdrucksformen indes religiös aufgeladen

45 Hellwig 1715, 35, ebenso 56. Schönheit und Tugend sah man auch dergestalt miteinander verknüpft, dass die Schönheit der Frau die Liebe des Mannes hervorrufe, dem man wiederum den Mann auf den Pfad der Tugend führe (vgl. Hellwig 1715, 31f.) - ein alter Topos, dem man zum Beispiel in der schönen Literatur des Mittelalters im Konzept der hohen Minne begegnet.

46 Hatte man um 1700 die Schönheit primär auf Gott zurückgeführt, so liessen spätere Autoren Gott meist aus dem Spiel oder doch im Hintergrund. Dafür wurden sie nicht müde, die Schönheit als ein Produkt der Natur und eines naturgemässen Lebens zu erklären.

47 Dazu allgemein Mertens 1968.

48 Die Vernünftigen Tadlerinnen (1725) 1, 24. Stück. Auch darin folgte man englischen Vorbildern. Vgl. Menck 1940, u.a. 42, 62, 91.

49 Um 1700 war Literatur mit Beispielen gelehrter Frauen recht verbreitet (vgl. u.a.Amaranthes 1715), auf die sich auch Hellwig berief und von «Frauenzimmern» meinte: «sie sind öffters zu hohen Dingen tüchtig, wenn sie von Jugend auff darzu gehalten worden» (Hellwig 1715, 23f.).

50 Reinhard 1756, 93. 
waren und manche Anklänge an den schon Jahrzehnte früher entfalteten Pietismus enthalten. Dies gilt für die schon erwähnte Schrift eines Anonymus über Gesundheits- und Schönheitspflege aus dem Jahr 1785. Seiner Magdalis als dem Inbegriff weiblicher Schönheit sprach er mehr moralische als körperliche Qualitäten zu bzw. spiegelten sich ihre Tugenden in vielen augenfälligen Reizen wider. Äussere und innere Schönheit waren nun viel stärker miteinander verwoben als in früheren Jahrzehnten. Hier nur ein kurzer Auszug: «[...] man sieht keinen Schmuck an ihr, als den [...] ihr tugendhaftes Herz mit eigenen Pinsel in das Gesicht mahlte». Unter ihrer «aufgeheiterten Stirne, wo man die Aufschrift der Vernunft liest, da spannet ein ränkefreues[!] Gemüth die niedlich beschattenden, gedrengten, wohlgetheilten Augenbraunen auf». Die Augen «lächeln [...] mit sanftmüthigen Blicken hervor. [...] Auf ihrer gewelbten, alabasternen Brust, da thronet standhafte Tugend.» ${ }^{51}$

In getragen-feierlichem Ton wird hier eine quasi-religiöse Verehrung und Verklärung weiblicher Schönheit vorgetragen, die stimmig in dem Ausruf endet: «O welch englisches Bild! - Höchster Grad menschlicher Schönheit! ${ }^{52}$ Doch sind die konkret benannten Tugenden kaum oder nicht eigentlich religiöser bzw. christlicher Natur. Am häufigsten ist die Sanftmut vertreten, was als Indiz für Rousseaus nicht zu unterschätzenden Einfluss auf einen erheblichen Teil der deutschen Bildungsschicht zu interpretieren ist, für den Sanftmut «die erste und wichtigste Eigenschaft einer Frau» war ${ }^{53}$. Ausser den eben erwähnten wurden in den deutschen Ratgebern Schamhaftigkeit, Ernst und Munterkeit, Witzigkeit, Nettigkeit, Freundlichkeit, Ordnungssinn, Häuslichkeit und an letzter Stelle schliesslich der Verstand genannt - Tugenden oder Eigenschaften, die im gesellschaftlichen Umgang angenehm sind oder eine tüchtige Hüterin des Hauswesens verheissen ${ }^{54}$.

Sieben Jahre später (1792) rief der Mediziner Georg Ernst Kletten Frauen dazu auf, Herz, Charakter und Geist auszubilden, und stellte klar, dass nicht etwa der «Geist des Nachdenkens» gemeint sei, denn dieser sei dem männlichen Geschlecht vorbehalten, sondern der «Geist der Empfindsamkeit» ${ }^{55}$. Die «wahre Empfindsamkeit» der weiblichen Seele, das geistige Leben im

51 Anonym 1785,39-42. Auch Kants Ideal der Schönheit in seiner Kritik der Urteilskraft (1790) verlangte den Ausdruck sittlicher Ideen. Vgl. Brockmeier 1988, 200f.

52 Anonym 1785, 42. Hier ist englisch im Sinne von engelhaft verwendet.

53 Nach Steinbrügge 1992, 71.

54 Bescheidenheit, Sanftheit und hausfrauliche Tugenden hatten die englischen Frühaufklärer schon in den Moralischen Wochenschriften propagiert. Vgl. Menck 1940, u.a. 91.

55 Kletten 1792, 194. 
Innern drücke sich in der Physiognomie, aber auch in der Bewegung aus ${ }^{56}$, lesen wir in der wenige Jahre später anonym erschienenen und mehrfach aufgelegten Schrift Anmuth und Schönheit des vielschreibenden Berliner Arztes und Apothekers Christian Gottfried Flittner. Die spätaufklärerischen Lehrmeister der Damenwelt stimmten überein, dass zur äusseren Erscheinung im allgemeinen und Gesichtsbildung im besonderen eine bestimmte verinnerlichte Haltung, nämlich ein ausserordentlich feinsinniges Gefühlsleben, hinzutreten müsse, das allein Grazie und Anmut und damit wahre Schönheit verleihe ${ }^{57}$. Im Vergleich zu diesen gesteigerten Anforderungen an weibliche Selbstzucht und Tugend erscheinen die traditionell-christlichen Tugendgebote als vergleichsweise leicht erfüllbar. So ist es nicht abwegig, von einem auch ärztlicherseits vorangetriebenen Domestikationsprogramm für Frauen zu sprechen ${ }^{58}$.

56 Anonym 1802 (= Flittner) (EA 1797), 25. Die Anmut war spätestens seit Friedrich Schillers Schrift Über Anmut und Würde (1793), in der er sie als «Schönheit der Bewegung» definierte (zitiert nach Ausg. 1971,71), eine zentrale Kategorie der ästhetischen Theorie. Dass Flittner aus ebendieser Abhandlung Schillers geschöpft hat (der seinerseits wiederum in manchem Punkt auf Kant rekurrierte), wird nicht nur aus dieser Übereinstimmung, sondern aus verschiedenen fast gleichlautenden Textstellen deutlich (vgl. etwa Anonym 1802, 1f. mit Schiller 1971, 74f.). - Zum Ideal der Anmut vgl. auch Spickernagel 1983.

57 Frauen der Bildungsschicht, auch die Frühfeministin Mary Wollstonecraft, scheinen nach den wenigen von Pia Schmid untersuchten Selbstzeugnissen die nicht nur in deutschen medizinischen Ratgebern, sondern in anderen europäischen Ländern und auf vielen anderen Wegen verbreiteten idealistischen Anforderungen an Schönheit und Tugend verinnerlicht zu haben. Sie sprechen von wahrer Anmut, würdiger Schönheit, schöner Seele und Tugend, erwähnen aber auch stolz ihre dem Zeitgeschmack entsprechenden körperlichen Merkmale, wie rundliche Arme oder kleine Hände. Vgl. Schmid 1992. - Zum französischen Entwurf der Frauen als des moralischen Geschlechts vgl. auch Steinbrügge 1992. - Als zartes und moralisches Geschlecht galt, wie unter anderem Londa Schiebinger herausstellt, den zeitgenössischen Medizinern nur die weisse Frau; im Kontrast zu ihr charakterisierten sie die schwarze Frau als robust, kräftig, lüstern und damit unmoralisch. Vgl. Schiebinger 1995b, 168; ferner Schiebinger 1995a, 229-244. Auch war nur die weisse Frau in der Lage, im Erröten ihre geistigmoralische Empfindsamkeit auszudrücken, wie Angela Rosenthal am Beispiel englischer Künstler und Publizisten, darunter auch Ärzte, kürzlich festgestellt hat (vgl. Rosenthal 2001). $\mathrm{Zu}$ dieser ausserordentlichen ästhetisch-moralischen Feinsinnigkeit passte kein sexuelles Begehren mehr, das allmählich aus dem zwischen 1730 und 1830 in Lexika vermittelten Frauenbild verschwand (vgl. Schmid/Weber 1986).

58 Einen frühen Beitrag zu den gewaltsamen Aspekten des Verhältnisses von Ärzten zum weiblichen Geschlecht lieferte Ute Frevert 1982. - Lieselotte Steinbrügge wehrt sich in ihrer ausführlichen Würdigung des Vorbildes Rousseau unter Verweis auf die in ihren Augen positiven Seiten seines Frauenbildes, wie Naturnähe, Instinkthaftigkeit, Sinn für Ästhetik und Moral, gegen sein negatives Patriarchen- und Frauendresseurimage in der jüngeren Forschung (vgl. Steinbrügge 1992, besonders 68f.). - Zweifellos waren die ärztlichen Tugendlehren nicht originell. Ärzte argumentierten gerade in nicht-medizinischen Fragen nicht anders als ihre nicht-ärztlichen Zeit- und Geschlechtsgenossen, die häusliche und gesellige Tugenden bei Frauen ebenfalls am meisten schätzten, wie etwa der heute noch allseits bekannte Adolph Freiherr von Knigge, der sich zu der Behauptung verstieg, Fieberfrost zu bekommen in Anwesenheit schöngeistiger und gelehrter Frauen (vgl. u.a. Honegger 1991, 96f.). 


\section{Zur ärztlichen Bewertung des Schminkens und der Schminke}

Die Tendenz zur Moralisierung zeigte sich im Jahrhundert der Aufklärung auch in der zunehmenden Verdammung von Putz und Schminke. Nach dem Muster der Vernünftigen Tadlerinnen, einer Moralischen Wochenschrift, die allenthalben Putzsucht, Eitelkeit, Simplicia, Koketterie und galantes Wesen der sogenannten Modeschwestern geisselte ${ }^{59}$, argumentierte wenig später (1732) auch die früheste populärmedizinische Zeitschrift für Frauen, Die vor sich und ihre Kinder Sorgfältigen Mütter, moralisch. Sie diskreditierte Eitelkeit, Ehrlosigkeit, Hoffart und Hochmut sich schminkender Frauen und verwarf das Schminken explizit als unstatthaften Versuch, den allmächtigen Schöpfer zu meistern ${ }^{60}$. Hinzu trat, allerdings erst an zweiter Stelle, das medizinische Argument, dass eine der wichtigsten Lebensarbeiten des menschlichen Leibes, die Ausdünstung durch die Schweisslöcher der Haut, durch das «Mahlwerck» verhindert werde, woraus zahllose Folgeschäden resultierten. Ebendiese Argumentation wurde seit der Jahrhundertmitte in die für Frauen verfassten Ratgeber übernommen, und die Unterdrückung der Ausdünstung wurde bis zum Ende des Untersuchungszeitraumes als ein besonderes Gefahrenmoment betrachtet.

Bezüglich der Inhaltsstoffe von Kosmetika äusserten die Verfasser von Schönheitsschriften bis um die Mitte des 18. Jahrhunderts nur ganz vereinzelt Bedenken. Bleiweiss war ein noch unkommentierter Bestandteil zahlreicher Schönheitsmittel. Auch Quecksilberpräparate wurden empfohlen. Vorsicht schien im Umgang mit Quecksilber nur dann geboten, so Vogel 1690 und später seine Kompilatoren, wenn es falsch präpariert sei ${ }^{61}$. Solches führe zu Kopf- und Gliederschmerzen, zerfressener Haut, Schwindel, Krämpfen und Lähme sowie zu schwarzen wackelnden Zähnen. Der Verfasser wähnte sich selbst jedoch im Besitz der richtigen Rezeptur, die er sodann bekanntgab. Allerdings riet er dazu, mit quecksilberhaltigen Schönheitspräparaten vorsichtig umzugehen und sie nicht in Augen, Nase und Mund zu bringen, nicht mehrmals täglich zu gebrauchen und zum Schutz der Zähne, Mandeln zu kauen und wieder auszuspeien.

Angesichts der Tatsache, dass die von Quecksilberpräparaten ausgehenden Gefahren schon lange bekannt waren und daher auch im zeitgenössischen fachinternen Schrifttum um 1700 thematisiert wurden, befremdet der im allgemeinen recht unbekümmerte Umgang mit diesen Stoffen in der medizinisch-kosmetischen Populärliteratur aus der ersten Hälfte des

59 Die Vernünftigen Tadlerinnen (1725-1726).

60 Die vor sich und ihre Kinder Sorgfältigen Mütter (1732), 13. Stück 105ff., hier 106.

61 Vgl. zum Folgenden Vogel 1690, 391. 
18. Jahrhunderts. Lediglich zwei frühe, nicht namentlich genannte Autoren wiesen auf die Schädlichkeit mancher kosmetischer Präparate, besonders der quecksilberhaltigen, hin $^{62}$. Gerade diese beiden Schriften aber wurden offenbar kaum rezipiert und blieben auch ohne Einfluss auf nachfolgende Autoren. Während der auch auf dem Kosmetiksektor sehr erfolgreiche Publizist Christoph Hellwig in seinem für medizinisches Fachpersonal geschriebenen Thesaurus pharmaceuticus vor Quecksilber warnte ${ }^{63}$, äusserte er als populärmedizinischer Autor keine Bedenken gegenüber der intendierten Laienleserschaft. Ob er und andere Ärzte die Gefahren bei Schminkpräparaten geringer einschätzten oder sich beim Lesepublikum nicht durch eine besonders vorsichtige und kritische Haltung unbeliebt machen wollten, muss vorläufig offenbleiben. Jedenfalls wurde erst seit der Mitte und verstärkt gegen Ende des 18. Jahrhunderts auch in populären Schönheitsratgebern als schädlich erkannte oder vermutete chemische Substanzen namhaft gemacht, neben Quecksilber und Blei vor allem weitere Metalle und Salze wie Alaun, Kobalt, Kupfer, Vitriol, Wismut und Zinn ${ }^{64}$.

Die in den Zeitschriften der Frühaufklärung vorgebrachten moralischen Argumente gegen die Verwendung von Schminke aber waren bis zum Ende des 18. Jahrhunderts schon häufig benutzt worden und daher abgegriffen. Ärzte begründeten ihre Schminkgegnerschaft nun vorwiegend medizinisch. Neben einer Vergrösserung von Erfahrung und Fachwissen mochte die Beschränkung auf rein fachliche Argumente auch besser zum beanspruchten Expertenstatus des Arztes passen. Deshalb wohl kam der Frankfurter Arzt Georg Friedrich Hoffmann 1791 zu dem Schluss: «Das Moralische dieser Gewohnheit [des Schminkens] zu bestimmen - gehört nicht für den Arzt.» ${ }^{65}$

62 Einer der beiden anonymisierten Verfasser liess sich ermitteln; ob der andere ein Mediziner war, bleibt offen. Es waren: J. L. H. 1694 sowie G[ottlob] S[chober] M[edicinae] D[octor] \& P[racticus] 1709. Vgl. auch die Warnungen vor roter Schminke eines anonymisierten Arztes, der noch sehr viele Rezepturen mit bleihaltigen Präparaten mitteilte. Vgl. D. F.1715, 9 sowie $16,29,34-36$ usw.

63 Vgl. beispielsweise Hellwigs Warnungen vor der Anwendung des Quecksilbers in seinem Thesaurus pharmaceuticus, Hellwig 1711, 209. Zum Quecksilber in der Untersuchungszeit vgl. Goldwater 1972, 231-243.

64 Eine Analyse der Debatte über die Schädlichkeit dieser Mittel würde hier zu weit führen. Sie soll an anderer Stelle vorgelegt werden.

65 Hoffmann 1791, 128. 


\section{Diätetik statt Kosmetik}

Wenn die alten Schönheitsideale keineswegs passé waren, das weibliche Geschlecht seit der Mitte des 18. Jahrhunderts sogar mehr und mehr als das «schöne Geschlecht» bezeichnet wurde, was boten publizierende Ärzte ihren Leserinnen an zur Herstellung oder Erhaltung der Schönheit? Wie schon im Zusammenhang mit den ärztlichen Selbstlegitimationen herausgestellt, kaum noch das, was im frühen 18. Jahrhundert die Gesundheits- und Schönheitsratgeber gefüllt hatte, nämlich Rezepte für Kosmetika wie Wasser und Essenzen, Öle, Pomaden, Salben und Pulver, Seifen und Parfüm, Pasten, Masken, Schminklappen und Pflaster ${ }^{66}$.

\section{Vor der Aufklärung: Die Herstellung von Schönheit durch Kosmetika}

Die Mittel der frühen Kosmetikliteratur dienten vornehmlich der Verbesserung und Verschönerung der Gesichtshaut. Die Autoren leisteten damit freilich nur einen beschränkten Beitrag zur Verwirklichung der in ihren Schriften vorgestellten Schönheitsideale. Sie klammerten manche Aspekte aus, die ältere lateinische Werke De decoratione und traditionelle Werke der Hausväterliteratur noch enthalten hatten oder die in zeitgenössischen medizinischen Dissertationen schon erörtert wurden, wie z.B. korrigierende chirurgische Eingriffe, diverse Massnahmen gegen Fettleibigkeit, Leibesübungen oder das Problem gesundheitsschädlicher Kleidung ${ }^{67}$. Sie lehnten sich vielmehr sehr eng an das Muster des traditionellen Arznei- oder Rezeptbuches an, beschränkten sich aber weitestgehend auf äusserlich anzuwendende Mittel $^{68}$, obwohl Christoph Hellwig beispielsweise ein Nota Bene seiner Bemerkung voranstellte, dass für eine gute Gesichtsfarbe «innerliche Sachen» eigentlich tauglicher seien, weil nämlich Blässe meist von einem erkalteten Magen herrühre ${ }^{69}$.

66 Allerdings gab es im späten 18. Jahrhundert ein Nebeneinander von modernen Aufklärungsschriften und traditionellen Rezeptbüchern, wie etwa dem Toiletten-Almanach (1788) und einem auf sehr alte Vorbilder zurückgehenden hauswirtschaftlichen Ratgeber (ca.1790), deren anonyme Autoren aber höchstwahrscheinlich keine Mediziner waren.

67 Die genannten Aspekte wurden zwar in einem populärmedizinischen Werk behandelt, das auch auf viele Probleme von Haut und Haaren einging, allerdings war es keine Schönheitsschrift, sondern Nicolas Andrys an Väter und Mütter gerichtetes Werk über die körperliche Erziehung des Kindes, das drei Jahre zuvor in der französischen Originalausgabe veröffentlicht worden war. Vgl. Andry 1744.

68 Wenn Schönheitseinbussen eine Folge innerer Krankheit waren, wurden auch diätetische Ratschläge erteilt, Besuche des Bades und/oder Arzneien empfohlen.

69 Hellwig 1715. - Innerlich wirkende Arzneien sollten lediglich dann verabreicht werden, wenn die Haut von Krankheit oder Verletzung gezeichnet war. Für solche Fälle wurden auch diätetische Ratschläge erteilt und/oder Besuche des Bades empfohlen. 
Bleiben wir bei Hellwig als dem meistgedruckten und meistgelesenen Schönheitsberater des frühen 18. Jahrhunderts. Er offerierte Mittel für die unterschiedlichsten Ansprüche und Probleme. Es finden sich sehr einfache Empfehlungen, etwa bei Muttermalen nüchternen Speichel aufzutragen oder durch das Kauen von Gewürznelken und Wacholderbeeren für wohlriechenden Atem zu sorgen, also Anweisungen zur völlig autarken Version der Selbsthilfe oder zum Gebrauch unbearbeiteter, für jedermann leicht zugänglicher Substanzen aus der freien oder kultivierten Natur. Andere vorgeschriebene Drogen waren nur aus Apotheken oder sogar nur aus Apotheken grösserer Orte mit umfangreicherem Sortiment zu beziehen. Manche Rezepte enthalten zahlreiche Ingredienzien aus allen drei Naturreichen, dem «chymischen», pflanzlichen und animalischen. Die Herstellung - vom Verrühren, Verschütteln, Erhitzen und Kochen bis hin zum Extrahieren, Mazerieren, Kalzinieren und Destillieren - umfasste zum Teil etliche Arbeitsschritte und erforderte in solchen Fällen komplexe Handlungsanweisungen.

Aus den rund 250 Schönheitsrezepturen seiner Heimligkeiten des Frauenzimmers sei hier zur Veranschaulichung eine herausgegriffen:

\begin{abstract}
Man nimmt schöne Holländische rohe Leinwand, tuncket solche etlichemahl in durchgeseihet Froschleich[!]-Pflaster, daß es allzeit wieder trocken werde, wenn es zuletzt gantz trocken, zerläßt man weiß Wachs ein halbes Pfund, Pomade 2. Loth, Wallrath (Sperm.cet.) 2. quentl. Campffer 7. gran, wohlgemischt, und den Campffer, zuletzt, wenn mans vom Feuer nimmt, drunter gethan; Mit dieser Mixtur wird die Leinwand, vermittelst eines grossen weichen Pinsels, auff einer Seite, nach dem Gesicht zu, überstrichen, und wenn es kalt und hart ist, mit einem andern reinen Tüchlein gerieben, und polirt. Solche Maßqven kühlen die rothen Gesichte, machen die Haut weich und delicat. ${ }^{70}$
\end{abstract}

Für eine populär- und volksmedizinische Tradition spricht hier wie in den meisten Fällen schon die äussere Form der Anweisung: die Wiedergabe in ganzen Sätzen und in deutscher Sprache statt in klassischer Rezeptformel in lateinischer Sprache und mit für Laien unverständlichen Apothekerzeichen. Hin und wieder finden sich aus Gründen der Vorsicht allerdings lateinische Rezepte, die nicht zum Nacharbeiten durch Laien gedacht waren, sondern vom Apotheker ausgefertigt werden sollten. Manchmal wurde ein Autor als Gewährsmann oder ein Buchtitel als Quelle genannt. Man bezog sich aber nicht nur auf Fachkollegen. Bürge für die Qualität eines Produktes konnte mindestens ebensogut eine namentlich genannte Adlige sein. Vereinzelt gaben Ärzte auch Wissen aus dem kosmetischen Alltag einfacher(er) Frauen weiter ${ }^{71}$.

70 Hellwig 1715, 60.

71 Vgl. Hellwig 1719, 34, z.B. auch D. F. 1715, 43, 63. 
Das vorliegende Rezept schreibt fünf Ingredienzien $\operatorname{vor}^{72}$, bei vier lag der Durchschnitt in dem von mir untersuchten Textkorpus Hellwigs. Nur vereinzelte Rezepturen enthielten mehr als ein Dutzend Drogen und blieben damit weit hinter dem zurück, was komplexe innere Arzneimittel der Zeit mit mehreren Dutzend Bestandteilen beinhalten konnten. Die aufgrund ihrer leichten Zugänglichkeit gern der Volksmedizin zugeordneten tierischen Drogen $^{73}$ dominieren im zitierten Rezept. Mit Kampfer weist es nur eine einzige pflanzliche Substanz auf, wohingegen Hellwig im allgemeinen pflanzliche Drogen recht häufig verwendete. Das Rezept enthält keine chemischmineralischen Drogen, die auch nur einen geringen Anteil (von zehn Prozent) seiner kosmetischen Drogen ausmachen, obwohl Hellwig seit seinem Studium in Jena in chemiatrischer Tradition stand. Für deren geringe Bedeutung im kosmetischen Bereich wird die Zielgruppe der Laien, vielleicht auch die volkstümliche Herkunft mancher Rezepte ausschlaggebend gewesen sein.

Der in der kosmetischen Literatur häufiger vorkommende Froschlaich wurde in diesem Fall sicher wegen seiner kühlenden Wirkung verwendet, in anderen Fällen aber wohl auch im Sinne der Signaturenlehre ${ }^{74}$, da er junges Leben enthielt und damit symbolisch für Jugendlichkeit stehen konnte. Unter den animalischen Stoffen waren überhaupt solche von jungen Lebewesen beliebt ${ }^{75}$. Vermutlich hatte man auch die Vorstellung, die Eigenschaft der Lebendigkeit zu übertragen, wenn ein Mittel unter Verwendung sogenannten Schlagwassers, also von Mühlrädern geschlagenen Wassers, präpariert oder dieses zusatzfrei in die Kopfhaut massiert wurde zum Anregen des Haarwuchses. Stärker noch fällt bei Hellwig und anderen die häufige Verwendung weisser Ingredienzien auf, die symbolisch für die erwünschte Reinheit und Weisse der Haut standen, wie etwa im Falle eines sogenannten Schminkwassers, zu bereiten aus weissen Semmelkrumen, Esels- oder Ziegenmilch, Eiweiss und einem «weiß jung Hünlein» ${ }^{76}$. Die Grenze zur Magie wurde mit vereinzelten Empfehlungen überschritten, etwa der Benutzung von Menstruationsblut oder der Berührung einer schadhaften Körperstelle mit dem Körperteil eines Toten ${ }^{77}$.

72 Allerdings ist die erwähnte Pomade ihrerseits ein aus mehreren Ingredienzien bestehendes Präparat, das man entweder selbst bereiten oder als Fertigprodukt in einer Apotheke kaufen konnte.

73 Vgl. Simon 1982, 238.

74 Allgemein zur Signaturenlehre der frühen Neuzeit Ohly 1999.

75 Zum Beispiel Spanferkel oder «eines jungen Knaben Harn» oder «Harn von einem jungen Mägdlein von 9. bis 10. Jahren». Hellwig 1715, 73, 70, 80.

76 Hellwig 1715, 88.

77 Vgl. Hellwig 1715, 98. 
Das kosmetische oder Therapieziel, im Falle des zitierten Rezeptes: die Vertreibung von Gesichtsröte, wurde oft angegeben, oder es ergab sich quasi von selbst, denn eine Pomade beispielsweise sollte die Haut glätten und weich machen, ein Rouge die Wangen und Lippen röten. Die Ursachen eines unerwünschten Symptoms blieben in aller Regel ausser Betracht. Es ging nicht um die Vermittlung gesundheitlichen Basiswissens, sondern um Anleitung zur Handlung. Dabei wurde den Adressatinnen grosse Handlungsfreiheit eingeräumt, denn Hellwig und andere ärztliche Autoren gaben oft mehrere Rezepte gegen einen Makel oder ein Leiden zur freien Wahl seitens der Leser an. In den Bezeichnungen mancher Kosmetika hoben sie mittels lobender Adjektive die Qualität des Produkts besonders hervor, wenn sie zum Beispiel ein Gesichtsreinigungsmittel ein «köstlich Wasser» nannten oder ein «gut Zahnpulver» ankündigten. Darüber hinaus trugen manche Mittel insofern Namen mit Werbecharakter, als sie nach ihrer Herkunft aus fremden Ländern oder grossen Städten benannt waren und damit an die Sehnsucht nach der märchenhaften und sprichwörtlichen weiten Welt appellierten oder aus der höfischen Welt stammten, wie die berühmten Schminkwässer der Grossherzogin von Florenz oder der Königin von Ungarn, und damit Identifikationsobjekte über die Standesgrenzen hinweg anboten ${ }^{78}$. Gerade diese werbende, bisweilen sogar marktschreierische Präsentation von Rezepten, zumal solchen mit fragwürdigen oder gesundheitsschädlichen Bestandteilen, machte späteren Medizin(historik)ern den Umgang mit ihren frühneuzeitlichen Standeskollegen zu einem heiklen Problem ${ }^{79}$.

Ergänzend sei angemerkt, dass es nicht nur bei der Vermittlung der Rezepturen blieb. In Einzelfällen nutzten Kosmetikbuchautoren ihre Publikationen auch als Möglichkeit zur Werbung für ihren Versandhandel mit selbstgefertigten Kosmetika, wie ein Anonymus im Jahr 1709, der 13 verschiedene Mittel offerierte, vom Haar stärkenden und dessen Wachstum befördernden Wasser, das Glas zu acht Groschen, bis zum köstlichen Balsam, das Döschen zu sechs Groschen ${ }^{80}$.

78 Das Kapitel «Naming Cosmetics» von Wykes-Joyce 1961,143-147, geht nur auf die in neuerer Zeit freilich besonders spektakulären Bezeichnungen für Duftwässer ein.

79 Vgl. dazu Sander 2002a.

80 Es war der anonymisiert publizierende Leipziger Arzt Gottlob Schober. Vgl. G. S. D. M. \& P. 1709. 
Den Zusammenhang von Schönheit und gesunder Lebensführung hatten die Autoren der älteren deutschsprachigen Schönheitsschriften nicht ausführlich diskutiert ${ }^{81}$. Die aus der Antike überlieferten sex res non naturales - (1) Luft, (2) Essen und Trinken, (3) Schlafen und Wachen, (4) Bewegung und Ruhe, (5) Zurückhaltung und Ausleerung und (6) Affekte - galten zwar als schönheitsrelevant, wurden aber nur beiläufig erwähnt. So auch von Christoph Hellwig:Vor kalter, die Haut angreifender Luft sollten Frauen sich hüten ${ }^{82}$. «In Essen und Trincken haben sich Weibsbilder, sonderlich schöne Damen, auch in acht zu nehmen, darmit sie nicht häßlich [...] werden.» ${ }^{83}$ Bei der Ernährung galt es vor allem, zu starke Reize, das heisst Schärfe, Säure, Süsse, zu vermeiden und mässig zu sein. Mässigkeit wurde auch für Wachen und Schlafen empfohlen. Menstruationsstörungen, aber auch zurückgehaltener weiblicher Samen machten krank und hässlich ${ }^{84}$. Obwohl man Affektstörungen für besonders schädlich hielt, wurden auch sie nur mit einer kurzen Bemerkung abgetan: $\ll \mathrm{N}[\mathrm{ota}] \mathrm{B}[\mathrm{ene}]$ Wider die Runtzeln ist nichts bessers, als daß man ohne Sorgen und Grillen lebet.» ${ }^{85}$

Die Diätetik trat aus ihrer randständigen Rolle in Sachen Schönheit ganz in den Vordergrund, als ein antiaristokratischer bürgerlicher Natürlichkeitskult, ein Nationalismus, der Kosmetik als etwas «Undeutsches» ablehnte ${ }^{86}$, eine verstärkte Sensibilisierung für die kosmetikbedingte Gesundheitsschäden und eine nicht zuletzt aus ärztlichen Professionalisierungsinteressen gespeiste Kritik an Selbstmedikation die Vermittlung von Schönheitsrezepten als falsch erscheinen liess. Typische Aufklärer veröffentlichten

81 Zwar enthalten etwa Hellwigs Heimligkeiten rund hundert Seiten diätetischer Ratschläge (vgl. Hellwig 1715, 222-320), aber nicht speziell für Frauen und ohne Rekurs auf die vorangegangenen Kapitel zur Schönheitspflege.

82 Vgl. Hellwig 1715, 58.

83 Hellwig 1715, 60, zum Folgenden 61.

84 Beides konnte zur damals vielbeachteten und besonders von Hellwig oft erörterten «Jungfern-Krankheit» führen, die die körperliche Schönheit zerrüttete, da ihr wesentliches Kennzeichen eine ungesund bleiche, sogar gelbliche oder grünliche Hautfarbe war, weshalb das Leiden auch «bleiche Sucht» oder «Miß-Farbe» genannt wurde (vgl. Hellwig 1715, 147-151 bzw. 163). Irene Hardach-Pinkes neuere Monographie über junge Mädchen zwischen 1750 und 1850 löst nicht ein, was der Titel Bleichsucht und Blütenträume verspricht (vgl. HardachPinke 2000); vgl. auch Siddall 1982.

85 Hellwig 1715, 66, in diesem Sinne auch 31 und 61.

86 Diese Seite des Naturismus hatte in Frankreich kein Äquivalent. Das ihm zugrundeliegende Denkmuster, Ungutes bzw. als ungut Empfundenes als fremd einzustufen, war in der Geschichte der Medizin nicht neu, wie bekanntermassen das Beispiel der sogenannten Franzosenkrankheit Syphilis zeigt. - Allgemein zu dem auch unter Gender-Aspekten interpretierbaren deutschen Nationalismus des 18. Jahrhunderts Herrmann u.a. 1996. 
kaum noch Rezepte für Schönheitsmittel, verbannten sie gern in den Anhang ihrer Gesundheitsbücher für Frauen, beschränkten sich dabei stärker auf einfache Hausmittel und warnten vor unsachgemässer Anwendung ${ }^{87}$. Nicht nur in Monographien, sondern auch unter den ärztlichen Beiträgen in Zeitschriften für Frauen avancierte die Diätetik zum zentralen Thema ${ }^{88}$. Ausser der Diätetik erachteten Ärzte der Spätaufklärung nurmehr allgemeines medizinisches Grundlagenwissen als geeignet, an (weibliche) Laien vermittelt zu werden, womit sie dem Konzept einer limitierten Aufklärung folgten ${ }^{89}$.

Ausserdem reduzierten sie die Frage weiblicher Schönheit auf das Problem der Gesundheit der Frau: «Denn nur Gesundheit ist jene reiche Quelle, die aus dem Goldmeere der schönheitsvollen Natur, alle Zierde wahrer Schönheit des Körpers daherführet.» ${ }^{90}$ Freilich eine verkürzte Sicht. Demgegenüber hatte etwa Hellwig 1715 noch geschrieben, es «kan Schönheit ohne Gesundheit nicht bestehen, aber wohl Gesundheit ohne Schönheit» ${ }^{91}$. Angesichts der aufklärerischen Gleichsetzung von Gesundheit und Schönheit konnten nun mit Fug und Recht Bücher, die allein der weiblichen Gesundheit gewidmet waren, auch das für Käuferinnen und Leserinnen wichtige Reizwort Schönheit im Titel führen, wie etwa die 1771 erschienenen Briefe eines Arztes an die Frauenzimmer, oder Regeln der Kunst die Gesundheit und Schönheit zu erhalten.

Vor allem propagiert wurde in diesen weitgehend diätetisch ausgerichteten Werken ${ }^{92}$ die Mässigkeit, die sogenannte «Mittelstraße» zwischen allen Extremen, die in den Monographien seit den 1780er Jahren auch mit Einfachheit, dem Charakteristikum des idealisierten Landlebens, assoziiert

87 Vgl. etwa Anonym 1771, 35.

88 Im Wochenblatt für's schöne Geschlecht beispielsweise war der wesentliche medizinische Beitrag eine schon im zweiten Stück des ersten Heftes einsetzende umfangreiche Serie zur «Frauenzimmer-Diätetik» (1979, S. 14f. und öfter) oder der umfangreiche diätetische Artikel über das «Temperament des Frauenzimmers» im Magazin für Frauenzimmer, 11. Stück [1782] 972-999. - Was ursprünglich auf Ärzte zurückgeht, gaben unter den oftmals anonym bleibenden Publizisten zweifellos auch Nicht-Ärzte in (Frauen-)Zeitschriften weiter.

89 Allgemein zur limitierten Aufklärung Meyer 1987.

90 Vgl. Anonym 1785, 57. - Obgleich in der zweiten Hälfte des 18. Jahrhunderts eine Onaniedebatte in den Printmedien geführt wurde, ausgelöst durch den seinerzeit wirkungsmächtigsten ärztlichen Volksaufklärer Tissot, der unter anderem den Verlust körperlicher Attraktivität als eine der «Folgen der Selbstbefleckung bey dem weiblichen Geschlechte» beschworen hatte (vgl. Tissots über ein Dutzend Mal in deutscher Übersetzung erschienene Onanieschrift, 1767 u.ö., benutzte Ausgabe: 1780, $485 \S 20$ ), wurde die Masturbation in den Schönheitsratgebern nicht erwähnt.

91 Hellwig 1715, 30.

$92 \mathrm{Zu}$ den Populärdiätetiken für Frauen im 18. Jahrhundert vgl. auch Sander 1998, 98-102; Borkowsky 1988. 
wurde, wie überhaupt der Stadt-Land-Gegensatz im populärmedizinischen Schrifttum aus den beiden letzten Dezennien des 18. Jahrhunderts gern thematisiert und das Landleben als mustergültig vorgestellt wurde ${ }^{93}$.

(1) Was die Luft anging, galt im allgemeinen sowohl zu kalte als auch zu heisse Luft, besonders aber der zu rasche Wechsel zwischen beidem als schädlich $^{94}$. Einzelne Autoren des ausgehenden 18. Jahrhunderts begannen allerdings, die kalte Luft als Teil eines Abhärtungsprogrammes positiv zu bewerten ${ }^{95}$. Obwohl Zugluft als Gefahrenquelle genannt wurde, begann man Wert auf die regelmässige Belüftung der Innenräume zu legen und sie der Luftverbesserung durch Aromatisierung, wozu schon die ältesten italienischen Kosmetikratgeber Rezepturen angeboten hatten, vorzuziehen. Die «dickere» städtische Luft galt wegen ihrer Verunreinigung als Gesundheitsgefahr, die Landluft hingegen als ausgesprochene Förderin der Schönheit ${ }^{96}$.

(2) Breiteren Raum nahm die Erörterung der Speisen und Getränke ein, wobei Mässigkeit oberstes Gebot war. Einig waren sich die Popularisierer weiterhin darüber, dass Frauen keine stark gewürzten und arzneilich wirkenden Speisen zu sich nehmen sollten, weder zu scharfe noch zu sauere, noch zu süsse, wobei Süssigkeit und Säure seit den 1780er Jahren als zahnschädigend beurteilt wurden. Süssigkeiten betrachtete man als besondere Verlockung für Frauen, denen Naschhaftigkeit als geschlechtstypische Untugend nachgesagt wurde, die sie mit Kindern gemeinsam hätten. Vor Essig und Kreide als gängigen innerlich wirkenden Schönheitsmitteln wurden die Leserinnen gewarnt ${ }^{97}$. Nur wenig, und zwar leichtverdauliche, einfache, nicht durch verschiedene Behandlungen verkünstelte Nahrung wurde angeraten und pflanzlicher den Vorzug vor (fäulniserzeugender) Fleischspeise gegeben ${ }^{98}$. Die rechte Art sich zu ernähren sei eher auf dem Land verbreitet,

93 Die Idealisierung der Landbevölkerung als schlicht und unschuldig steht in unaufgelöstem Widerspruch zur Kritik am Landvolk, das (ärztliche) Aufklärer doch zugleich und insbesondere als Träger des unbedingt auszurottenden Aberglaubens wahrnahmen. Vgl. etwa Böning 1990 oder Masel 1997, darin beispielsweise den Tadel des bayerischen Landvolks aus einem Volkskalender von 1804 (Masel 1997, 207-217). Zu diesem Spannungsverhältnis vgl. auch Sadowsky 1998.

94 In diesem Zusammenhang ging es um Fragen wie Abkühlung nach erhitzendem Tanzen oder den modisch entblössten Busen, wo medizinische und moralische Urteile eng miteinander verflochten wurden. Der Bekleidungsfrage, besonders den Schnürbrüsten, haben aufklärerische Ärzte bekanntlich ganze Monographien gewidmet. Vgl. dazu etwa Junker/ Stille 1988; Sahmland 1992 sowie Sahmland unveröff. Ms.

95 Vgl. etwa Siede 1797, 161, in diesem Falle ein Nicht-Arzt, der die aktuelle Strömung popularisierte.

96 Anonym 1785 sowie Kletten 1792.

97 Vgl. Hoffmann 1743, 10 und 13.

$98 \mathrm{Vgl}$. Anonym 1785, 67ff.-70. 
wo selbst «das genaue Kauen» noch üblicher sei als in der Stadt ${ }^{99}$. In einem grösseren Bezugsrahmen betrachtete der nämliche Autor die deutsche Esskultur mit ihrem insgesamt eher einfachen und bescheidenen Geschmack als ursprünglicher, natürlicher, unverdorbener und mit gesünderem Urteil begabt als andere Esskulturen - wobei das unausgesprochene negative Gegenbild zweifellos Frankreich war ${ }^{100}$. Hatte man in den 1740er Jahren noch feste Regeln und Ordnung in der Ernährung besonders betont, so stand mit der modischen Zivilisationskritik des späten 18. Jahrhunderts die Idee des Naturgemässen und Einfachen im Vordergrund.

Im gesamten Untersuchungszeitraum wurde beanstandet, dass viele Frauen zu wenig trinken würden, obwohl andererseits auch ausgiebige «Theetrank»-Nachmittage als mögliche Ursache des gefährlichen «weißen Flusses» beanstandet wurden, womit sich die Abschaffung einer Form weiblicher Geselligkeit medizinisch rechtfertigen liess ${ }^{101}$. Wenn sich Ärzte gegen übertriebenen Genuss der Heissgetränke Tee, Kaffee und Schokolade wandten, ging es nur zum Teil um die Inhaltsstoffe, etwa die arzneiliche Wirkung des Kaffees. Für manche war die Eigenschaft Wärme der eigentliche Stein des Anstosses, weil sie den Körper erschlaffe und speziell den Zähnen schade. Deshalb empfahl ein Arzt sogar, die üblichen Heissgetränke wie er selbst «hübsch kalt zu trinken» ${ }^{102}$. Auch gegen Alkoholmissbrauch seitens «Wein- und Bier-Schwester[n] und Brandteweins-Bulle[n]» ${ }^{103}$ hatten sich Ärzte schon in den frühen Schriften grundsätzlich ausgesprochen ${ }^{104}$. Doch erschien die Neigung zum Alkoholkonsum mit den rigider werdenden ärztlichen Vorstellungen von Weiblichkeit in der Zeit der Spätaufklärung immer weniger vereinbar, so dass aufgrund einer Alkoholabhängigkeit, die als üble Gewohnheit oder Laster und noch nicht als Krankheit galt, den Betroffenen sogar die Zugehörigkeit zum weiblichen Geschlecht abge-

99 Anonym 1785, 65; in diesem Sinne auch Anonym 1795, 22.

100 Vgl. Anonym 1795, 63 und 76. Demgegenüber war man im Corpus hippocraticum (Über die Anfänge der Medizin) von einem Geschichtsmodell des zivilisatorischen Fortschritts auch in der Ernährung ausgegangen, von der allmählichen Ersetzung roher, grober und schlecht bekömmlicher Nahrung durch bekömmliche Speisen und Zubereitungsarten. Nach Fischer, unveröff. Ms., 6.

101 Anonym 1741,23f.

102 Anonym 1795, 22.

103 Hellwig 1715, 61. Gemeint waren offenbar Branntweinbuhlen, also Liebhaberinnen hochprozentiger Getränke.

104 Über den Gebrauch des Weines im Fall von Schwäche und Kränklichkeit gab es keine einhellige Meinung. Manche hielten ihn für ein geeignetes Stärkungsmittel (vgl. Anonym 1785, 119), andere erachteten ihn gerade bei Kränklichkeit für nicht zuträglich (vgl. Essich 1787, 132). 
sprochen wurde ${ }^{105}$. Als Durstlöscher für Gesunde hatten Ärzte in der ersten Hälfte des 18. Jahrhunderts dünnes Bier und verdünnten Wein noch ausdrücklich befürwortet, ebenso Limonaden. Kaltes Wasser, das in früheren Jahrhunderten als schädliches Getränk für Frauen aufgrund des angenommenen weiblichen kalt-feuchten Temperamentes gegolten hatte ${ }^{106}$, wurde im gesamten Untersuchungszeitraum für gut befunden, doch bekam es im Laufe des 18. Jahrhunderts einen fast kultischen Status, als Inbegriff reiner und unverdorbener Natur. Das Wassertrinken sollte eine «Hauptsorge der Schönen» sein ${ }^{107}$. Neben ihm konnte im späten 18. Jahrhundert kein anderes Getränk mehr bestehen. Empfohlen wurde reines Brunnenwasser. Mineralwässer aber sollte man wegen ihrer arzneilichen Kräfte nur auf Anraten eines Arztes trinken ${ }^{108}$.

(3) In bezug auf Wachen und Schlafen ergaben sich im Jahrhundert der Aufklärung keine neuen Aspekte für die Belehrung der weiblichen Leserschaft. Ärzte sprachen sich durchweg für frühes Schlafengehen und frühes Aufstehen aus. Sofern konkrete Uhrzeiten angegeben wurden, bedeutete das eine Nachtruhe von ca. 21 Uhr bis fünf Uhr morgens ${ }^{109}$. Dieser gegenüber dem heute Üblichen versetzte Wach-Schlaf-Rhythmus entsprach den Gepflogenheiten einer überwiegend agrarischen Gesellschaft. Für Frauen der gehobenen und höheren Schichten hatte die Vorgabe dieses Rhythmus zugleich eine erzieherische oder sozialdisziplinierende Funktion, indem er Abendgesellschaften und Vergnügungen weitgehend ausschloss und bürgerliche Tugenden wie Arbeitsamkeit und Fleiss zu befördern suchte. Auffallend ist, dass Frauen ausdrücklich eine eher kurze Nachtruhe nahegelegt wurde. Dem sogenannten «schwachen Geschlecht» sollte weniger durch Ruhe und Schlaf geholfen werden als durch mässige Reizung und Belastung, vor allem durch Hausarbeit ${ }^{110}$, was zum Aspekt Bewegung und Ruhe überleitet.

105 Hoffmann 1791, 37-39, hier 38f. Aber schon im frühen 16. Jahrhundert hatte Johann von Schwarzenberg, der Verfasser der Peinlichen Halsgerichtsordnung, eine Unordnung der Geschlechterabgrenzungen und -beziehungen befürchtet, wenn sich Frauen mit gesteigertem Alkoholgenuss ein männliches Vorrecht anmassten. Nach Frank 1998, 197f. Eine medizinische Kritik weiblichen Alkoholkonsums im 18. Jahrhundert enthielt die 1737 in Halle unter Michael Alberti von Johannes Christian Göhrs verteidigte Dissertation.

106 Vgl. Diepgen 1963, 211.

107 Anonym 1785, 85-89, hier 88.

108 Vgl. Hoffmann 1791, 28.

109 Vgl. Hoffmann 1743, 70.

110 Die Betätigung im Haushalt hatte schon im Mittelalter wegen ihrer wärmenden, aber nicht überlastenden Wirkung als gesundheitsfördernd für Frauen gegolten. Vgl. Diepgen 1963, 208. 
(4) Wenngleich sich im Verlauf des 18. Jahrhunderts in den Ratgebern die Ansicht des berühmten Schweizer Arztes und Aufklärers Samuel Auguste Tissot mehr und mehr durchsetzte, dass ein Mangel an Bewegung der Gesundheit und Schönheit abträglich sei, wurde doch auch vor zuviel Bewegung gewarnt und nur Reden, Lachen, Singen, Musizieren sowie Spazieren oder Fahren empfohlen. Sportliche Übungen im engeren Sinne spielten kaum je eine Rolle ${ }^{111}$. Als typisch weibliche Lustbarkeit war das Tanzen ein in medizinischen wie pädagogischen Schriften oft diskutierter Gegenstand ${ }^{112}$. Die meisten Ärzte stuften es als gefährlich, einzelne sogar als tödlich ein ${ }^{113}$. Auch in diesem Punkt hat es den Anschein, als ob der Aspekt, Frauen zu domestizieren, ausschlaggebend für ärztliche Stellungnahmen gewesen sei.

(5) Zu den im Prozess der Zivilisation (Norbert Elias) zunehmend tabuisierten Bereichen gehörten die Evakuationen des menschlichen Körpers, was sich auch an den populärmedizinischen Schriften unseres Untersuchungszeitraumes beobachten lässt: «Hm! - Nein!», so liess der anonyme Verfasser des 1785 erschienenen Buches über Gesundheits- und Schönheitspflege den betreffenden Abschnitt beginnen, um dann zu betonen, dass das Thema nicht stillschweigend übergangen werden dürfe ${ }^{114}$. Die neue Peinlichkeit erstreckte sich zum Teil selbst auf Informationen über Ausdünstungen durch die Poren; die Warnungen vor der Gefahr einer Unterdrückung der Ausdünstung durch Schminke sind oben schon erwähnt worden. Vornehmlich aber wurden die Menstruation und ihre Störungen unter den Stichworten Zurückhaltung und Ausleerung in den an Frauen adressierten Schriften abgehandelt. In diesem

111 Nur Melchior Adam Weikard sprach kurz vor der Wende zum 19. Jahrhundert in seiner allerdings für Damen und Herren gedachten Toilettenlektüre explizit von Leibesübungen (vgl. Weikard 1797, 20). Dazu zählte er etwa das Reiten. Von solch eifrigen Körperübungen, die laut Oribasios die Menstruation positiv beeinflussen sollten (vgl. Diepgen 1963, 212), war sonst aber nicht die Rede, da dergleichen die Frau mit ihrer angeblich schwachen Konstitution zu überfordern schien. - In seiner auf vier medizinischen Quellengattungen (Dissertationen, Diätetiken, moral- und staatshygienischen Abhandlungen) beruhenden Untersuchung der Einstellung deutscher Ärzte des 18. Jahrhunderts zu Leibesübungen (ohne geschlechtsspezifischen Bezug) kam Herbert Hänel ebenfalls zu dem Schluss, dass die Meinungen weit auseinandergingen, von der Ablehnung bis zur Befürwortung körperlicher Übungen (vgl. Hänel 1972, 17-27).

112 Auch die für junge Mädchen verfassten pädagogischen Schriften dieser Zeit schärften immer wieder die Schädlichkeit übermässiger Bewegungen und des Tanzens, namentlich des Walzens, ein (vgl.Pellatz 1999,73f.).-Am Beginn der Neuzeit hatten solche diätetischen Anweisungen speziell für Schwangere gegolten, siehe etwa Jacob Rueffs «Vnderweisung wie sich die Schwangeren Frauwen halten sollen» (vgl. Rueff 1563, 245-249, hier 246).

113 Allein der anonyme Verfasser der Briefe eines Arztes an die Frauenzimmer erklärte 1771 tägliches Tanzen für erheiternd und belebend und als gut für Muskeln, Gelenke und Haltung (vgl. Anonym 1771, 138), wie es auch im frühen 18. Jahrhundert für sehr gesund befunden worden war (vgl. D. F. 1715, 15). Essich liess das Tanzen gelten mit dem Zusatz: «Es versteht sich aber, dass sie nicht auf Sibillenart tanzen müssen» (Essich 1790, 49).

114 Anonym 1785, 209. 
Zusammenhang stellten Ärzte seit der Mitte des 18. Jahrhunderts auch die Schnürbrüste an den Pranger, welche sowohl Menstruation als auch Nierenund Darmtätigkeit beeinträchtigten. Auf recht einsamem Posten stand Johann Gottfried Essich, ein eher als Popularisierer denn als Aufklärer im engeren Wortsinne zu bezeichnender vielschreibender Augsburger Arzt, wenn er unter Berufung auf die französische Damenwelt sich nachhaltig für die regelmässige Anwendung von Klistieren als wahren Schönheitsmitteln stark machte ${ }^{115}$, und zwar zu einer Zeit (1787), in der Ärzte hierzulande im allgemeinen eher antifranzösisch orientiert waren und zumeist deutsches Wesen an sich selbst genesen lassen wollten - auch wenn sie im übrigen Rousseausches Gedankengut verbreiteten.

(6) Im Hinblick auf sämtliche Affekte, auch auf positive, wie Freude, Hoffnung oder Liebe, galt Mässigkeit und Zurückhaltung als oberstes Gebot, das gerade Frauen besonders zu berücksichtigen hätten, da sie aufgrund geschlechtsspezifischer Veranlagung, nämlich durch ihre viel reizbareren Nerven, wie man im späten 18. Jahrhundert meinte, grundsätzlich sehr viel gefühlsbetonter reagierten als Männer. Alle starken Affekte und Leidenschaften aber schadeten der Gesundheit und damit mittelbar der Schönheit, machten aber auch unmittelbar hässlich, indem sie die Gesichtszüge verzerrten.

(7) In keinem Populärwerk fehlte seit Beginn der siebziger Jahre des 18. Jahrhunderts ein Kapitel über die Reinlichkeit, die nun als neues Element zu den sex res non naturales der traditionellen diätetischen Lehre hinzutrat ${ }^{116}$. Zwar finden sich in den frühesten deutschen medizinisch-kosmetischen Ratgebern für Frauen zahlreiche Rezepte für Seifen. Doch waren die vor allem für die Hände gedacht, wie schon manche Rezeptüberschriften erkennen lassen, die sich indes nicht auf den reinigenden, sondern oft auf den im engeren Sinne kosmetischen Aspekt beziehen: «Eine wohlriechende Seiffe zu den Händen», «Weiche Seiffe, schöne Hände zumachen»"17. Zur Reinigung oder Behandlung des Gesichts hingegen wurde häufig für abends ein kosmetisches Destillat, für morgens entweder frisches Wasser oder ein Absud empfohlen. Die in der höfischen Welt Frankreichs ausgeprägte Wasserscheu, die dort favorisierte Reinigung der Haut mit einem trockenen weissen Tuch war in Deutschland nicht üblich ${ }^{118}$.

115 Vgl. Essich 1787, 173f. Zur Empfehlung der Klistiere als Schönheitsmittel seitens französischer Ärzte vgl. auch Perrot 1984, 42f.

116 Sie bezog sich nicht allein auf den Körper, sondern auch auf Kleidung, Wäsche und - wie oben angedeutet - auf reine (Stuben-)Luft.

117 Hellwig 1715, 77f.

118 Vgl. Vigarellos Kapitel über die «trockene Toilette» (Vigarello 1988, 26-30). Eine Ausnahme in Deutschland ist ein Anonymus 1774, 7. 
Parallel zur Aufwertung des Naturprodukts Wassers als Getränk im Zuge der Aufklärung gewann das kalte oder kühle Wasser als äusserliches Mittel zur Reinigung sowie zur Stärkung und Abhärtung des Körpers grosse Bedeutung, auch mit dem Argument, dass es schön mache, während warmes Wasser die Haut «erschlappe» ${ }^{119}$. So sollte das Badewasser besser lauwarm sein als warm ${ }^{120}$. Die Ganzkörperhygiene war bis zur Moderne kein Thema der Ratgeber. Welche Körperteile im einzelnen und wie häufig gewaschen werden sollten, wurde auch gegen Ende unserer Untersuchungszeit kaum je erwähnt ${ }^{121}$. Wenn ein anonymer Autor von der morgendlichen und abendlichen Waschung sprach, mag sich dies wohl nur auf das Gesicht bezogen haben $^{122}$. Ein anderer empfahl tägliche Gesichtsreinigung, «öfters» Fusswaschungen, im übrigen aber Waschungen «zu verschiedenen Zeiten» ${ }^{123}$, was die Leserinnen nach Gusto auslegen konnten. Christoph Wilhelm Hufeland forderte wenig später in seinem nur nebenhin Schönheitsfragen berührenden Bestseller Makrobiotik indes ausdrücklich die tägliche Reinigung des ganzen Körpers und einmal wöchentlich ein laues $\mathrm{Bad}^{124}$. Mit solchen Forderungen, die unsere gegenwärtigen Hygienestandards vorwegnahmen, erschien er manchem Zeitgenossen vielleicht nicht weniger wirklichkeitsfremd als jener sentimentalische städtische Arzt der deutschen Spätaufklärung, der vorbildliche Hygiene zum Charakteristikum des Landlebens erklärte und vor seinem geistigen Auge - und wohl nur dort - des Landmannes Tochter und die Schäferin sich allmorgens am frischen Silberquell waschen $\operatorname{sah}^{125}$.

Alle diätetischen Lehren, die in der zweiten Hälfte des 18. Jahrhunderts doch das Fundament der medizinischen Aufklärung des weiblichen Lesepublikums bildeten und Garanten für Gesundheit und Schönheit sein sollten, stiessen aber schon vor 1800 auf tiefes Misstrauen in den eigenen Reihen.

119 Anonym 1785, 258; in diesem Sinne aber auch schon D. F. 1715, $13 f$.

120 Hoffmann hatte in seiner 1743 ins Deutsche übersetzten Jungfer-Diät schon vor zu warmen Bädern gewarnt (vgl. Hoffmann 1743, 36). Vereinzelt wurde später die Temperatur des Waschwassers von der jeweiligen individuellen Beschaffenheit der Haut abhängig gemacht (vgl. Anonym 1781, 93-95).

121 Zur Hygiene vgl. vor allem Frey 1997; ausserdem Löneke/Spieker 1996, deren Sammelband sich allerdings auf das 19. und 20. Jahrhundert bezieht; zu den abweichenden französischen Verhältnissen vgl. Vigarello 1988.

122 Vgl. Anonym 1795, 11. Er sah in diesen Waschungen vor allem ein Abhärtungsmittel, befürchtete aber, dass die Haut davon rauh und hart werden könne.

123 Anonym 1785, 250.

124 Hufeland 1798, Kapitel XI: «Reinlichkeit und Hautkultur», 168-181, hier 172. Zu der um 1800 so hohen Stellenwert gewinnenden Sauberkeit und Hygiene sowie zu ihrer Verquickung mit Moral in der Gesundheitserziehung vgl. Labisch 1985 und 1986.

125 Anonym 1785, 253. 
So wollte der Nürnberger Arzt Johann Karl Osterhausen in seiner Kritik der medizinischen Populärschriften allein die Reinlichkeit anerkennen, weil sie als Trieb zur menschlichen und tierischen Natur gehöre. «Diätetik aber ruht vielleicht unter allen medicinischen Scienzen auf den schwankendsten Gründen, die mehrsten ihrer Lehren sind Meinungen», beanstandete er $1798^{126}$. Die Ge- und Verbote in den sogenannten sechs nicht natürlichen Dingen könne man streichen und durch die eine Grundregel der Mässigkeit sowie eine genaue Beobachtung der individuellen körperlichen Gegebenheiten ersetzen. Das aber hätte in bezug auf Frauen bedeutet, sie vom Korsett des medizinisch begründeten und ärztlicherseits verbreiteten Geschlechtsstereotyps zu befreien, ein Unterfangen, das auch im wenig später anbrechenden neuen Jahrhundert noch keineswegs realisiert werden sollte.

\section{Resümee und Ausblick}

Wenn ich im folgenden die Ergebnisse der Bestandsaufnahme zur deutschen kosmetischen oder Schönheitsliteratur der frühen Neuzeit und ihrer Veränderung unter dem Einfluss der Aufklärung zusammenfasse, sollen die zentralen Fragen der Wiener Gespräche $e^{127}$ nach der Etablierung von Normen und nach Grenzziehungen besonders berücksichtigt werden:

1. Die in den Schönheitsratgebern vermittelten, sich auf den weiblichen Körper beziehenden ästhetischen Normen erwiesen sich über die Zeiten hinweg als erstaunlich beständig. Zierlichkeit als das wesentliche Charakteristikum brachte eine deutliche, im Verlauf der Jahrzehnte eher zunehmende Abgrenzung gegenüber dem männlichen Geschlecht zum Ausdruck, die zugleich die Frau als schwaches und dem Kind ähnliches Wesen erscheinen liess. Die zwar häufig, jedoch nicht im Zusammenhang mit Schönheitsidealen vorgebrachte Kritik der geschnürten schlanken Taille war weniger ein Bekenntnis zu der im allgemeinen gepriesenen Natürlichkeit als eine Konsequenz der weiblichen Bestimmung zur Mutterschaft.

2. Wie bei der äusseren Schönheit der Frau wurde auch ärztlicherseits hinsichtlich ihrer inneren Qualitäten auf den Differenzen zwischen den

126 Osterhausen 1798,43f., 64-66. Er hatte dabei besonders die physische Erziehung der Kinder im Visier, formulierte seine Kritik aber, wie aus dem Zitat schon ersichtlich, in einer ins Allgemeine gehenden Form.

127 Vgl. die allererste Anmerkung. 
Geschlechtern insistiert, sogar mit besonderem Nachdruck in der Zeit der Spätaufklärung. Weiblichkeit wurde dann mit besonderer Tugendhaftigkeit, namentlich mit Sanftmut assoziiert, während traditionelle christliche Tugenden einen deutlichen Bedeutungsverlust erlitten hatten. Das neue Konstrukt eines ausserordentlich verfeinerten weiblichen Seelenlebens liess die Geschlechter noch weiter auseinander driften.

3. In der Populärliteratur zeichnete sich im Verlauf des 18. Jahrhunderts eine deutliche Veränderung ärztlichen Selbstverständnisses ab. Vom «Galanteriearzt» und «Leibdiener der Schönheit», der die Interessen und Bedürfnisse seiner Kundschaft zu befriedigen suchte, entwickelte sich der Arzt zum Experten für Gesundheit, die in der Spätaufklärung mit Schönheit weitgehend gleichgesetzt wurde. Als Gesundheitsexperte musste der Arzt seine kosmetische Beratungstätigkeit, die sich zu einer kosmetikkritischen gewandelt hatte, nicht weiter rechtfertigen.

4. Das veränderte Selbstverständnis, der Wille zur Professionalisierung implizierten auch eine Grenzziehung gegenüber den Patientinnen. Ärzte der Spätaufklärung machten einen Führungsanspruch der (männlichen) Fachleute gegenüber den (weiblichen) medizinischen Laien geltend, womit sie sehr entschieden eine Umkehr der früheren Hierarchie anstrebten. Als Autoren gaben viele im letzten Drittel des 18. Jahrhunderts kaum noch oder gar nicht mehr Anleitungen zur Selbstmedikation in Form von kosmetischen Rezepten, die sie ehemals sogar von Frauen übernommen hatten, sondern versuchten aufgrund ihres diätetischen Fachwissens das sogenannte «schöne Geschlecht» zu erziehen und zu formen, oft im Sinne einer Domestizierung. Bei allen Gesundheitsproblemen, die immer auch eine Gefahr der Einbusse von Schönheit bedeuteten, sollten ärztliche Fachleute konsultiert werden.

5. Ihren Expertenstatus bekundeten Ärzte in einer seit der Jahrhundertmitte in den Monographien oftmals formulierten und zunehmend fachlich begründeten Kritik an kosmetischen Präparaten. Normierung und Grenzziehung gingen aber häufig über das rein Fachliche hinaus: Eine nationale Grenzziehung erfolgte gegenüber Frankreich durch die Aufwertung des (vermeintlich) Deutschen, vor allem deutscher Einfachheit, was zugleich eine soziale und ideologische Abgrenzung war, nämlich der bürgerlichen Welt und ihres Natürlichkeits- und Tugendideals, von der höfisch-aristokratischen Welt und ihrer Künstlichkeit.

6. Um ihren bürgerlichen Normen, die sie zu implementieren suchten, einen topographischen und sozialen Ort in der eigenen Gegenwart zuzuweisen und ihre Ideale nicht nur als Teil eines verlorenen goldenen Zeitalters zu betrauern, beschworen einzelne Ärzte der Spätaufklärung in der Nach- 
folge Rousseaus schwärmerisch und idealistisch das Landleben als Hort eines gesund-tugendhaften Lebens.

7. In der vorliegenden Studie zur populären Schönheits- und Kosmetikliteratur deutscher Provenienz ist der gravierende Wandel beschrieben worden, der sich von den voraufklärerischen Schriften des späten 17. und frühen 18. Jahrhunderts hin zu den aufklärerischen des späten 18. Jahrhunderts abzeichnete. Es war ein Wandel oder besser Umschwung von der These, der recht undifferenzierten Propagierung von Kosmetika, zur Antithese, einer harschen Kritik an Schönheitsmitteln und ihren Benutzerinnen, verbunden mit dem Versuch, die Kosmetik ganz und gar durch Diätetik zu ersetzen. Diese Antithese wurde allerdings nur von einer einzigen Generation von Publizisten vertreten. Wenige Jahre vor der Wende zum 19. Jahrhundert begann eine zweite Generation von Spätaufklärern die Synthese der beiden extremen Positionen einzuleiten, die im Rahmen des vorliegenden Beitrages aber nicht näher untersucht werden konnte. Nur so viel sei angedeutet: Diese jüngere Generation bemühte sich um einen Kompromiss zwischen weiblichen und ärztlichen Interessen, indem sie Schönheitsmittel nicht in Bausch und Bogen verdammte, aber den Aspekt der Gesundheitsgefährdung durch Kosmetika mit reflektierte, sich um medizinisch Unbedenkliches bemühte und die Themen Körperhygiene und Diätetik in ihre Schönheitsschriften mit aufnahm ${ }^{128}$.

\section{Quellen}

Alberti, Michael (Präs.)/Johannes Christianus Göhrs (Resp.), Dissertatio inauguralis medica de ebrietate foeminarum (Halae Magdeburgicae 1737)

Amaranthes (= Gottlieb Siegmund Corvinus), Nutzbares, galantes und curiöses FrauenzimmerLexicon (Leipzig 1715)

Andry, Nicolas, Orthopädie, oder die Kunst, Bey den Kindern die Ungestalt des Leibes zu verhüten und zu verbessern (Berlin 1744)

Anonym (= Christian Gottfried Flittner), Anmuth und Schönheit aus den Misterien der Natur für ledige und verheirathete Frauenzimmer (Berlin 1802, EA 1797)

Anonym, Arzt des Frauenzimmers (Leipzig 1773)

Anonym (= Konrad Anton Zwierlein), Der Arzt für Liebhaber der Schönheit (Heidelberg 1781)

Anonym, Briefe eines Arztes an die Frauenzimmer (Leipzig 1771)

Anonym, «Frauenzimmer-Diätetik», in: Wochenblatt für's schöne Geschlecht (1779) 2. Stück $14 \mathrm{f}$. u. öfter (ND Leipzig 1967)

Anonym, Geheime und nach vielfältiger Erfahrung bewährte rare Kunststücke für alles Frauenzimmer [...] (Frankfurt/Leipzig [ca. 1790])

Anonym, Die Gesundheits- und Schönheitspflege für Frauenzimmer (Wien 1785)

Anonym, Leib-Diener der Schönheit (Leipzig 1747)

Anonym, Mittel zur Erhaltung und Beförderung der Schönheit und Gesundheit besonders für junge Herren und Damen v. e. verstorbenen Arzte (s.l.1795)

128 Vgl. dazu Sander 2002b. 
Anonym, Neu-entdeckte Geheimnisse von der Schönheit der Damen (Leipzig 1704)

Anonym, Neuestes Handbuch für junges Frauenzimmer, 2. Th. (Hamburg 1774)

Anonym, Neue woleingerichtete Frauenzimmerapotheke oder gründliche Anweisung, wie ein jedes Frauenzimmer in allen ihren kränklichen und geheimen Zufällen ihr eigener Arzt seyn könne (Hamburg 1741)

Anonym (= Johann Christoph Heppe), Toiletten-Almanach oder Handbuch für Frauenzimmer [...], hg. v. e. Verehrer geschikter Frauenzimmer (Leipzig/Linz 1788)

Art. «Schönheit», in: Amaranthes (= Gottlieb Siegmund Corvinus), Nutzbares, galantes und curiöses Frauenzimmer-Lexicon (Leipzig 1715) 1756-1758

Art. «Schönheit des Frauenzimmers», in: Universal Lexicon aller Wissenschaften und Künste, Bd. 35 (Halle/Leipzig: Zedler 1743) 822-830

Baldinger, Ernst, Neue Arzeneien wider die medicinischen Vorurtheile, Bd. 1 (Langensalza 1768)

D. F., Außerleßene Frauen-Zimmer-Medicin, das ist:Viele und gewisse Mittel, Welche, die äusserlichen Gliedmassen und schöne Gestalt in seinem Stand erhalten [...] (Leipzig 1715)

Essich, Johann Gottfried, Medicinisches Taschenbuch für Deutschlands Töchter (Augsburg 1787)

- Kleines medizinisches Taschenbuch für's ledige schöne Geschlecht (neue Aufl. Wien/Leipzig 1790)

Greneille, François de, Frauenzimmer-Belustigung [...]. Übersetzt durch den Unglückseligen [= Johann Wilhelm von Stubenberg] (Nürnberg 1653)

G. S. M. D. \& P. (= Gottlob Schober Medicinae Doctor \& Practicus), Kurtze / doch ausführliche Beschreibung Pharmacopoeae cosmeticae [...] (s.l. [vermutl. Leipzig] 1709)

Hartmann, Anton Theodor, Über die Ideale weiblicher Schönheit bei den Morgenländern [...] (Düsseldorf 1798)

Hellwig, Christoph, Thesaurus pharmaceuticus [...] (Leipzig 1711)

- Entdeckte Heimligkeiten Des Frauenzimmers [...] (Frankfurt/Leipzig 1715)

Hellwig, Christoph von, Armer Leute Sicherer und Geschwinder Hauß-Medicus [...] (2. verm. Aufl. Frankfurt/Leipzig 1719)

Hoffmann, Friedrich, Gründliche Abhandlung von der Jungfer-Diät oder wie sich eine Jungfer zuverhalten Wenn sie gesund und wohlgestalt will bleiben (Wittenberg/Zerbst 1743)

Hoffmann, Georg Friedrich, Wie können Frauenzimmer frohe Mütter gesunder Kinder werden, und selbst dabei gesund und schön bleiben? (2. verm. u. verb. Aufl. Frankfurt/Leipzig 1791)

Hufeland, Christoph Wilhelm, Makrobiotik oder Die Kunst das menschliche Leben zu verlängern (Jena 1798, EA 1796)

Hygea. Eine heilkundige Zeitschrift dem weiblichen Geschlechte von Stande vorzüglich gewidmet (1793)

J. L. H., Neu Eröffnete Schminck-Apothek [...] (s.1.1694)

Kletten, Georg Ernst, Versuch einer Geschichte des Verschönerungstriebes im weiblichen Geschlechte (Gotha 1792)

Magazin für Frauenzimmer (1782ff.)

Oelßner, Gottlieb, Philosophisch-Moralisch- und Medicinische Betrachtung Ueber mancherley Zur Hoffarth und Schönheit hervorgesuchte, schädliche Zwang-Mittel, junger und erwachsener Leute, beyderley Geschlechtes (Breßlau/Leipzig 1754)

Osterhausen, Johann Karl, Ueber medicinische Aufklärung, Bd. 1 (Zürich 1798)

Reinhard, Christian Tobias Ephraim, Satyrische Abhandlung von den Krankheiten der Frauenspersonen, welche sie sich durch ihren Putz und Aufzug zuziehen (Glogau/Leipzig 1756)

Rueff, Jacob, Hebammen Buch / Daraus man alle Heimligkeiten de $\beta$ Weiblichen Geschlechts erlehrnen [...] könne (Franckfort am Mayn 1563)

Schiller, Friedrich, «Über Anmut und Würde», in: Friedrich Schiller, Kallias oder über die Schönheit. Über Anmut und Würde (Stuttgart 1971), 67-151 (EA 1793)

Siede, J. C., Versuch eines Leitfadens für Anstand, Solidität, Reiz, Grazie und weibliche Schönheit (Dessau 1797)

Soemmerring, Samuel Thomas, «Über die Schädlichkeit der Schnürbrüste», in: Christian Gotthilf von Salzmann (Hrsg.), Über die Schädlichkeit der Schnürbrüste, 2 Preisschriften [...] (Leipzig 1788)

Struve, Carl Wilhelm Friedrich, Gesund- und Schönheits-Pflichten Zur Erbauung des andern Geschlechts wissenschaftlich erwiesen (Dresden 1754) 
Tissot, S[amuel] A[uguste] D[avid], «Die Onanie, oder Abhandlung über die Krankheiten, die von der Selbstbefleckung herrühren [...]», in: S. A. D. Tissot, Sämmtliche zur Arzneykunst gehörige Schriften, nach den neuesten [...] Originalausgaben aus dem Franz. u. Lat. übers. u. mit einigen Anm. begl. v. Johann Christian Kerstens. 2. Th. (Leipzig 1780) 419-678 (EA 1767)

Tissot, Wilhelm (= Friedrich A. Kritzinger), Besonderen Entdeckungen von der Schönheit der Frauenzimmer (s.1.1788, EA 1777)

Die Vernünftigen Tadlerinnen, hg. v. Johann Christoph Gottsched (1725-1726, ND Hildesheim u.a. 1993)

Vogel, Tobias, Curieuser Haut-Diener, Vorstellend Der Menschlichen Haut Schönheit und Heßligkeit. Worbey zu deren Erhaltung und Verbesserung dienliche Mittel vorgeschlagen werden (Leipzig 1690)

- Spiegel der Menschlichen Schönheit [...] (Leipzig 1687)

Die vor sich und ihre Kinder Sorgfältigen Mütter (1732)

Weikard, Melchior Adam, Toilettenlektüre für Damen und Herren in Rücksicht auf ihre Gesundheit (Frankfurt 1797) 1-118

W[oyt], J[ohann] J[akob], Der Neu-verfertigte Galanterie-Artzt (Dresden 1699)

\section{Literatur}

Angeloglou, Maggie, A History of Make-up (London 1970)

Audoin, Martial, Art. «Grenailles (François)», in: Nouvelle Biographie Génerale [...], Bd. 21 (Paris 1857) 912f.

Bleker, Johanna, «Die Frau als Weib: Sex und Gender in der Medizingeschichte», in: Christoph Meinel/Monika Renneberg (Hrsg.), Geschlechterverhältnisse in Medizin, Naturwissenschaft und Technik (Bassum/Stuttgart 1996) 15-29

Böning, Holger, «Medizinische Volksaufklärung und Öffentlichkeit». In: IASL 15,1 (1990) 1-92

Borkowsky, Maya, Ärztliche Vorschriften zur Schwangerschaftshygiene im 19. Jahrhundert unter Berücksichtigung einiger Aspekte der Diätetik für Gebärende, Wöchnerinnen und Stillende (Zürich 1988)

Brockmeier, Peter, «Vom verliebten Haß über den erquickenden Verdruß zum schmerzlichen Genuß. Weibliche Schönheit in Texten von Dante bis de Sade», in: Theo Stemmler (Hrsg.), Schöne Frauen - schöne Männer: Literarische Schönheitsbeschreibungen (Tübingen 1988) 199-233

Corson, Richard, Fashions in Makeup, from Ancient to Modern Times (London ${ }^{41997)}$

Dane, Gesa, «Die heilsame Toilette»: Kosmetik und Bildung in Goethes «Der Mann von funfzig Jahren» (Göttingen 1994)

Daniel, Ute, Kompendium der Kulturgeschichte. Theorien, Praxis, Schlüsselwörter (Frankfurt 2001)

Diepgen, Paul, Frau und Frauenheilkunde in der Kultur des Mittelalters (Stuttgart 1963)

Dumont, Franz u.a. (Hrsg.), Moguntia medica. Das medizinische Mainz vom Mittelalter bis ins 20. Jahrhundert (Wiesbaden 2002)

Fischer, Klaus-Dietrich, Essen und Trinken (unveröff. Ms.)

Frank, Michael, «Trunkene Männer und nüchterne Frauen. Zur Gefährdung von Geschlechterrollen durch Alkohol in der Frühen Neuzeit», in: Martin Dinges (Hrsg.), Hausväter, Priester, Kastraten. Zur Konstruktion von Männlichkeit in Spätmittelalter und früher Neuzeit (Göttingen 1998) 187-212

Frevert, Ute, «Frauen und Ärzte im späten 18. und frühen 19. Jahrhundert - zur Sozialgeschichte eines Gewaltverhältnisses», in: Annette Kuhn/Johann Geyer-Kordesch (Hrsg.), Frauen in der Geschichte III (Düsseldorf 1982) 177-210

Frey, Manuel, Der reinliche Bürger. Entstehung und Verbreitung bürgerlicher Tugenden in Deutschland, 1760-1860 (Göttingen 1997)

Gadebusch Bondio, Mariacarla, «Heilung des Körpers und Pflege der Schönheit anhand eines venezianischen Rezeptars des 16. Jahrhunderts», in: Hans-Uwe Lammel (Hrsg.), Kranksein in der Zeit. Referate des Rostocker Medizin- und Wissenschaftshistorikertreffens 1995 (Rostock 1996) 57-78 
Gilman, Sander L., Making the Body Beautiful. A cultural history of aesthetic surgery (Princeton 1999 u. 2001)

Goldwater, Leonard J., Mercury. A History of Quicksilver (Baltimore, Maryland 1972)

Hänel, Herbert, Deutsche Ärzte des 18. Jahrhunderts über Leibesübungen (Frankfurt 1972)

Hardach-Pinke, Irene, Bleichsucht und Blütenträume. Junge Mädchen 1750-1850 (Frankfurt 2000)

Hausen, Karin, «Die Polarisierung der 〈Geschlechtscharaktere»», in: Werner Conze (Hrsg.), Sozialgeschichte der Familie in der Neuzeit Europas (Stuttgart 1976) 363-393

Herrmann, Hans Peter u.a., Machtphantasie Deutschland. Nationalismus, Männlichkeit und Fremdenhass im Vaterlandsdiskurs deutscher Schriftsteller des 18. Jahrhunderts (Frankfurt 1996)

Honegger, Claudia, Die Ordnung der Geschlechter. Die Wissenschaften vom Menschen und das Weib 1750-1850 (Frankfurt a. M. 1991)

Junker, Almut/Eva Stille, Zur Geschichte der Unterwäsche. Ausstellungskatalog (Frankfurt 21988)

Labisch, Alfons, «Die soziale Konstruktion der «Gesundheit〉 und des 〈Homo hygienicus〉. Zur Soziogenese eines sozialen Gutes», in: Österr. Zs. f. Soziologie 10 (1985) 60-81

- «Hygiene ist Moral - Moral ist Hygiene〉. Soziale Disziplinierung durch Ärzte und Medizin», in: Christoph Sachße/Florian Tennstedt (Hrsg.), Soziale Sicherheit und soziale Disziplinierung. Beiträge zu einer historischen Theorie der Sozialpolitik (Frankfurt 1986) 265-285

Laqueur, Thomas, Auf den Leib geschrieben. Die Inszenierung der Geschlechter von der Antike bis Freud (Frankfurt u.a. 1992)

Lerner, Gerda, «Welchen Platz nehmen Frauen in der Geschichte ein? Alte Definitionen und neue Aufgaben», in: Elisabeth List (Hrsg.), Denkverhältnisse, Feminismus und Kritik (Frankfurt 1989) 334-352

Löneke, Regina/Ira Spieker (Hrsg.), Reinliche Leiber - schmutzige Geschäfte. Körperhygiene und Reinlichkeitsvorstellungen in zwei Jahrhunderten (Göttingen 1996)

Lohse-Jasper, Renate, Die Farben der Schönheit. Eine Kulturgeschichte der Schminkkunst (Hildesheim 2000)

Marwick, Arthur, Beauty in History. Society, Politics and Personal Appearance c. 1500 to the Present (London 1988)

Masel, Katharina, Kalender und Volksaufklärung in Bayern. Zur Entwicklung des Kalenderwesens 1750 bis 1830 (St. Ottilien 1997)

Meinel, Christoph/Monika Renneberg (Hrsg.), Geschlechterverhältnisse in Medizin, Naturwissenschaft und Technik (Bassum/Stuttgart 1996)

Menck, Ursula, Die Auffassung der Frau in den frühen moralischen Wochenschriften (Diss. Hamburg 1940)

Mertens, Wolfgang, Die Botschaft der Tugend. Die Aufklärung im Spiegel der Moralischen Wochenschriften (Stuttgart 1968)

Meyer, Robert, «Limitierte Aufklärung. Untersuchungen zum bürgerlichen Kulturbewußtsein im ausgehenden 18. und beginnenden 19. Jahrhundert», in: Hans Erich Bödeker/Ulrich Herrmann (Hrsg.), Über den Prozeß der Aufklärung in Deutschland. Personen, Institutionen und Medien (Göttingen 1987) 139-200

Mosse, George L., Das Bild des Mannes. Zur Konstruktion der modernen Männlichkeit. Aus dem Amerikanischen von Tatjana Kruse (Frankfurt am Main/Wien 1997)

Most, G. W. u.a., Art. «Schöne, das», in: Historisches Wörterbuch der Philosophie, hg. v. Joachim Ritter/Karlfried Gründer, Bd. 8 (Basel 1992) 1343-1385

Ohly, Friedrich, Zur Signaturenlehre der Frühen Neuzeit. Bemerkungen zur mittelalterlichen Vorgeschichte und zur Eigenart einer epochalen Denkform in Wissenschaft, Literatur und Kunst. Aus dem Nachlaß hg. v. Uwe Ruberg/Dietmar Peil (Stuttgart/Leipzig 1999)

Pellatz, Susanne, Körperbilder in Mädchenratgebern. Pubertätslektüre zur Zeit der Formierung bürgerlicher Kultur (Weinheim u.a. 1999)

Perrot, Philippe, Le travail des apparences ou les transformations du corps féminin XVIII $-X I X^{e}$ siècle (Paris 1984)

Pinset, Jacques/Yvonne Deslandres, Histoire des soins de beauté (Paris 1960)

Rang, Brita, «Zur Geschichte des dualistischen Denkens über Mann und Frau. Kritische Anmerkungen zu den Thesen von Karin Hausen zur Herausbildung der Geschlechtscharaktere 
im 18. und 19. Jahrhundert», in: Jutta Dalhoff u.a. (Hrsg.), Frauenmacht in der Geschichte. Beiträge des Historikerinnentreffens 1985 zur Frauengeschichtsforschung (Düsseldorf 1986) 194-204

Richter, Klaus, Die Herkunft des Schönen. Grundzüge der evolutionären Ästhetik (Mainz 1999)

Ridder, Paul, Schön und gesund. Das Bild des Körpers in der Geschichte (Kassel 1996)

Ritter, Joachim, Art. «Ästhetik, ästhetisch», in: Historisches Wörterbuch der Philosophie, hg. v. Joachim Ritter/Karlfried Gründer, Bd. 1 (Basel 1971) 555-580

Rosenthal, Angela, «Die Kunst des Errötens. Zur Kosmetik rassischer Differenz», in: Herbert Uerlings u.a. (Hrsg.), Das Subjekt und die Anderen. Interkulturalität und Geschlechterdifferenz vom 18. Jahrhundert bis zur Gegenwart (Berlin 2001) 95-117

Sadowsky, Thorsten, «Agrarromantik und Großstadtkritik im Zeitalter der Aufklärung», in: Anne Conrad u.a. (Hrsg.), Das Volk im Visier der Aufklärung. Studien zur Popularisierung der Aufklärung im späten 18. Jahrhundert (Hamburg 1998) 103-120

Sahmland, Irmtraut, «Kleider machen krank. Mediziner des 18./19. Jahrhunderts als Modekritiker», in: Die Waage 31 (1992) 101-108

- Samuel Thomas Soemmerring: Über die Wirkungen der Schnürbrüste (unveröff. Ms.)

Sander, Sabine, «〈Von den sonderbahren Geheimnüssen des Frauen-Zimmers〉 zur 〈Schwachheit des schönen Geschlechts». Frauen in der Populärmedizin des 18. Jahrhunderts», in: Thomas Schnalke/Claudia Wiesemann (Hrsg.), Die Grenzen des Anderen. Medizin aus postmoderner Perspektive (Köln 1998) 75-120

- «Der Arzt und die Kosmetik - Zerrbilder im Spiegel der deutschen Medizinhistoriographie», in: Med. Hist. J. 37 (2002a) 265-300

- « Vergebens unterdrückt man in unserer modernen Welt die Schminke». Ärztliche Beiträge aus Mainz zur Kosmetik-Diskussion im späten 18. Jahrhundert», in: Franz Dumont u.a. (Hrsg.), Moguntia medica. Das medizinische Mainz vom Mittelalter bis ins 20. Jahrhundert (Wiesbaden 2002b) 251-261

Schiebinger, Londa, Am Busen der Natur. Erkenntnis und Geschlecht in den Anfängen der Wissenschaft (Stuttgart 1995a)

- «Die Anatomie der Differenz. «Rasse» und Geschlecht in der Wissenschaft», in: Iris Bubenik-Bauer/Ute Schalz-Laurenze (Hrsg.), Frauen in der Aufklärung. «...ihr werten Frauenzimmer, auf!» (Frankfurt 1995b) 155-172

- Feminism and the Body (Oxford 2000a)

- "Skeletons in the Closet: The First Illustrations of the Female Skeleton in EighteenthCentury Anatomy", in: Londa Schiebinger (ed.), Feminism and the Body (Oxford 2000b) $25-57$

Schmid, Pia/Christina Weber, «Von der «wohlgeordneten Liebe〉 und der «so eigenen Wollust des Geschlechtes`. Zur Diskussion weiblichen Begehrens zwischen 1730 und 1830», in: Jutta Dalhoff u.a.(Hrsg.), Frauenmacht in der Geschichte. Beiträge des Historikerinnentreffens 1985 zur Frauengeschichtsforschung (Düsseldorf 1986) 150-165

Schmid, Pia, «Zwischen Wollust und Tugend. Schönheit im weiblichen Diskurs um 1800», in: Farideh Akashe Böhme (Hrsg.), Reflexionen vor dem Spiegel (Frankfurt 1992) 50-63

Schuster, Martin, «Gesichtsschönheit: Begriff, Geschichte und Merkmale», in: Manfred Hassebrauck/Reiner Niketta (Hrsg.), Physische Attraktivität (Göttingen 1993) 11-28

Schwarzkopf, Jutta u.a., «Geschlechtergeschichte. Von der Nische in den Mainstream», in: Zs. f. Geschichtswiss. 50 (2002) 485-504

Siddall, A. Clair, "Chlorosis - Etiology reconsidered", in: Bull. Hist. Med. 56 (1982) 254-260

Simon, Gabriele, Kosmetische Präparate vom 16. bis 19. Jahrhundert (Diss. Braunschweig 1983)

Somogyi,Tamar, Die Schejnen und die Prosten. Untersuchung zum Schönheitsideal der Ostjuden in Bezug auf Körper und Kleidung unter besonderer Berücksichtigung des Chassidismus (Diss. Berlin 1982)

Spickernagel, Ellen, «Zur Anmut erzogen - Weibliche Körpersprache im 18. Jahrhundert», in: Ilse Brehmer u.a. (Hrsg.), Frauen in der Geschichte IV (Düsseldorf 1983) 305-319

Steinbrügge, Lieselotte, Das moralische Geschlecht. Theorien und moralische Entwürfe über die Natur der Frau in der französischen Aufklärung (Stuttgart ${ }^{21992)}$ 
Stolzenberg-Bader, Edith, «Weibliche Schwäche - männliche Stärke. Das Kulturbild der Frau in medizinischen und anatomischen Abhandlungen um die Wende des 18. zum 19. Jahrhundert», in: Jochen Martin/Renate Zoepffel (Hrsg.), Aufgabe, Rollen und Träume von Mann und Frau, Teilbd. 2 (Freiburg 1989) 751-818

Verspohl, Franz-Joachim, «Die Entdeckung der Schönheit des Körpers - Von seiner maßästhetischen Normierung zu seiner bewegten Darstellung», in: Richard van Dülmen (Hrsg.), Erfindung des Menschen. Schöpfungsträume und Körperbilder 1500-2000 (Wien u.a. 1998) $139-157$

Vigarello, Georges, Wasser und Seife, Puder und Parfüm. Geschichte der Körperhygiene seit dem Mittelalter (Frankfurt u.a. 1988)

Wykes-Joyce, Max, Cosmetics and Adornment. Ancient and Contemporary Usage (London 1961) 